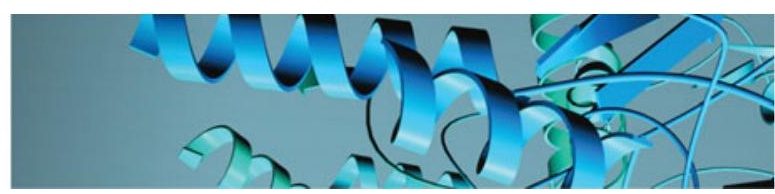

REVIEW

\title{
The evidence for open and closed exocytosis as the primary release mechanism
}

\author{
Lin Ren ${ }^{1}$, Lisa J. Mellander ${ }^{2}$, Jacqueline Keighron ${ }^{1}$, Ann-Sofie Cans ${ }^{1}$, Michael E. Kurczy ${ }^{1}$, Irina Svir $^{3}$, \\ Alexander Oleinick ${ }^{3}$, Christian Amatore ${ }^{3}$ and Andrew G. Ewing ${ }^{1,2 *}$ \\ ${ }^{1}$ Department of Chemistry and Chemical Engineering, Chalmers University of Technology, 41296 Gothenburg, Sweden \\ ${ }^{2}$ Department of Chemistry and Chemical Biology, University of Gothenburg, 41296 Gothenburg, Sweden \\ ${ }^{3}$ Département de Chimie, Ecole Normale Supérieure-PSL Research University, Sorbonne Universités-UPMC Univ. Paris 06, CNRS UMR 8640 PASTEUR, \\ 24 rue Lhomond, 75005 Paris, France
}

Quarterly Reviews of Biophysics (2016), 49, e12, page 1 of 27 doi:10.1017/S0033583516000081

Abstract. Exocytosis is the fundamental process by which cells communicate with each other. The events that lead up to the fusion of a vesicle loaded with chemical messenger with the cell membrane were the subject of a Nobel Prize in 2013. However, the processes occurring after the initial formation of a fusion pore are very much still in debate. The release of chemical messenger has traditionally been thought to occur through full distention of the vesicle membrane, hence assuming exocytosis to be all or none. In contrast to the all or none hypothesis, here we discuss the evidence that during exocytosis the vesicle-membrane pore opens to release only a portion of the transmitter content during exocytosis and then close again. This open and closed exocytosis is distinct from kiss-and-run exocytosis, in that it appears to be the main content released during regular exocytosis. The evidence for this partial release via open and closed exocytosis is presented considering primarily the quantitative evidence obtained with amperometry.

\section{An overview of exocytosis and endocytosis 2}

2. Techniques to monitor exocytosis 5

2.1. Patch clamp 6

2.2. Amperometry 6

2.3. Patch amperometry 7

2.4. Cyclic voltammetry 7

2.5. Fluorescence microscopy imaging 8

\section{Traditional models of exocytosis 9}

4. Evidence where partial release during exocytosis appears to be dominant 11

4.1. The total content of a secretory vesicle is greater than the amount released 11

4.2. The majority of exocytotic events are followed by immediate endocytosis 12

4.3. Full distention of the vesicle may not be necessary for quantal release 13

4.4. A flickering fusion pore might regulate quantal release and vesicle recycling 15

4.5. Nanometer-sized pores can be seen as 'feet' associated with amperometric spikes

* Author for Correspondence: A. G. Ewing, Department of Chemistry and Chemical Engineering, Chalmers University of Technology, 41296 Gothenburg, Sweden. E-mail: andrewe@chalmers.se 
5. Factors affecting the extent of partial release during individual events 18

5.1. Lipid composition of the plasma membrane 18

5.2. Mechanically applied pressure to the membrane 19

5.3. Osmolality 19

5.4. Temperature 20

5.5. External $\mathrm{pH} 20$

6. Regulation of open and closed exocytosis 21

6.1. Evidence that dynamin regulates the opening of the fusion pore 21

6.2. Evidence that actin regulates the closing of the fusion pore 21

7. Summary of the evidence for open and closed exocytosis 22

7.1. The majority of exocytotic events are followed by immediate endocytosis 22

7.2. The total content of a secretory vesicle is greater than the amount released in PC12 cells 23

7.3. Release appears to be only a fraction of vesicle content in mammalian brains 23

7.4. Impact cytometry of adrenal cell vesicles shows a significantly larger catecholamine content then that released 23

7.5. Impact cytometry has been used to measure vesicle content in live cells and this quantity is greater than the amount released 23

7.6. Full distention of the vesicle is not necessary for the measured amperometric current during exocytotic release 23

7.7. Fast changes in cell environment alter the amount of messenger released 23

7.8. Patch amperometry measurements reveal a greater amount of release 23

7.9. Postspike 'feet' are observed with an amperometric spike 23

Acknowledgements 24

References 24

\section{An overview of exocytosis and endocytosis}

Neurons transmit information by converting electrical signals to chemical signals through a process called exocytosis. In the resting stage, transmitters are stored in small organelles of nearly uniform size and shape, the synaptic vesicles. When an action potential arrives in the nerve terminal, the membrane depolarizes and voltage-gated $\mathrm{Ca}^{2+}$ channels open. The subsequent $\mathrm{Ca}^{2+}$ influx triggers exocytosis of synaptic vesicles, resulting in the release of neurotransmitter into the synaptic gap between two neighboring neurons (Jahn \& Sudhof, 1994; Lawson et al. 1977; Steyer et al. 1997; Tsai et al. 2008; Wu et al. 2014; Zucker, 1996). Exocytosis has become a key area for research providing understanding of the molecular mechanisms that control the process of chemical communication between neurons. Using electrophysiological measurements at the frog neuromuscular junction, it was first discovered by Katz in the 1950s, that subsequent to an action potential, a train of potentials with small and constant size occurred at the postsynaptic membrane. These quantized mini endplate potentials led to the hypothesis that chemical messengers are released in quanta (Del Castillo \& Katz, 1954, 1957; Fatt \& Katz, 1952). This resulted in the identification of the synaptic vesicle as the storage compartment for these molecules. The observation of the presence of synaptic vesicles by electron microscopy in the presynaptic terminals later suggested that these compartments release their content of chemical messengers into the synaptic gap between neighboring neurons as a part of their communication. Following this release called exocytosis, the signaling molecules then diffuse across the synaptic gap and bind to specific receptors on the postsynaptic membrane. The mechanistic process of exocytosis involves the docking of neurotransmitter-filled vesicles by vesicle-SNARE proteins present in the vesicle membrane to recognize and bind to target-SNAREs in the presynaptic plasma membrane (Mohrmann et al. 2010). The vesicles reside in this docked position until an action potential triggers $\mathrm{Ca}^{2+}$ channels in the presynaptic terminal to open a fusion pore. The formation of this fusion pore allows neurotransmitter molecules to exit the pore as it expands. The general theory of exocytosis suggests that it is an all or none process and that the synaptic vesicle membrane and proteins are then rapidly retrieved and reutilized for the formation of new synaptic vesicles, a process called endocytosis (Foley et al. 2011; Wu et al. 2014). These vesicles are reloaded with transmitter for another round of exocytosis, a cycle that is repeated many times and can be studied in nerve terminals that have no connection with the neuronal cell body. Thus, the presynaptic compartment consists of an autonomous unit that contains all components required for repetitive exocytosis and membrane recycling (Foley et al. 2011; Jin et al. 2008; Wu et al. 2014). To get more insight, transmission electron microscopy (TEM) methods have been widely used in the last decades to visualize the ultrastructure of the cell and acquire information about the small-sized vesicle compartments in cells, especially during exocytosis process. With this highresolution imaging technique, it has been possible to determine the vesicle size (typically $50-800 \mathrm{~nm}$ in diameter) and 

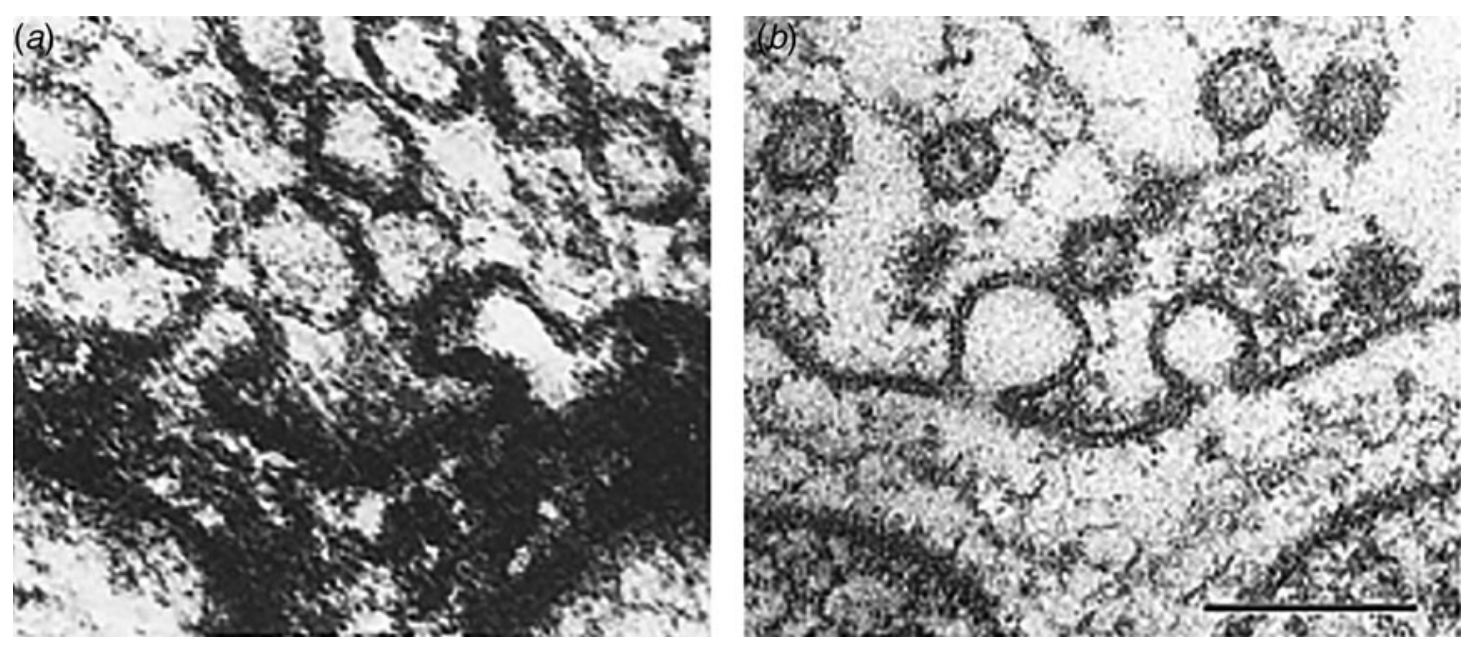

Fig. 1. Synaptic vesicle exocytosis as it first appeared in chemically fixed frog neuromuscular junctions circa 1970 (a), from Couteaux \& Pecot-Dechavassine (1970), and as it appeared in quick-frozen ones circa $1980(b)$, from Heuser \& Reese (1981). Scale bar $=0 \cdot 1 \mu \mathrm{m}$.

abundance in single neurons, as well as vesicle morphological changes that occur during the exocytosis process. The first clear-cut images of synaptic vesicle exocytosis were obtained by Counteaux and his co-workers in the 1970s (Fig. 1). As impressive as these images are, the fact that they were obtained somewhat serendipitously, i.e. without stimulation of the nerve, left the problem of correlating synaptic vesicle discharge with synaptic activity unresolved (Couteaux \& Pecot-Dechavassine, 1970; Heuser \& Reese, 1981). However, a break-through in this regard came with R. Miledi's discovery that ionic $\mathrm{La}^{3+} \mathrm{mys}^{-}$ teriously stimulates massive quantal discharge from the frog neuromuscular junction (Miledi, 1966). With this treatment, Miledi and Heuser were able to demonstrate an unequivocal reduction in synaptic vesicle numbers after exhaustive quantal discharge.

The TEM images showed the $\mathrm{La}^{3+}$-induced depletion of synaptic vesicles and their replacement by large vacant endosomes during prolonged transmitter release at the frog neuromuscular junction (Fig. 2a), compared with a control nerve terminal (Fig. 2b) (Heuser \& Miledi, 1971). This morphological change was the first indication that synaptic vesicle membrane might recycle via standard mechanisms of endocytosis. Later, the omega shape of vesicles observed in TEM images from cells stimulated to undergo exocytosis was suggested to correspond to fused vesicles with a fusion pore connection to the plasma membrane. Under low level of stimulation, B. Ceccarelli freeze-fractured a frog neuromuscular prepared in the presence of horseradish peroxidase (HRP). He was able to catch synaptic vesicles connected through a narrow neck to the synaptic cleft, taking up HRP through the fusion pore. Vesicles were seen in association with the plasma membrane and, in rare instances, these vesicles were captured in the process of forming an 'omega' shape as they were fusing with the plasma membrane (Fernández-Peruchena et al. 2005; Holt et al. 2004; Kops et al. 1990; Sesaki \& Ogihara, 1997). More interestingly, some synaptic vesicles were already labeled inside the presynaptic terminal. Ceccarelli proposed that under low stimulation conditions vesicles will fuse transiently with the presynaptic membrane, release some content through a fusion pore, and detach and move inside the presynaptic membrane awaiting a new round of exocytosis. These experiments indicated that synaptic vesicles might not necessarily collapse at the active zone with the presynaptic membrane to release neurotransmitters. This raises an important issue about the mechanism involved in the formation of the fusion pore and the subsequent dynamics that determine the mode for exocytosis after pore opening, which is still highly contested.

There are two main modes of exocytosis, which have been characterized as 'full fusion' and 'kiss-and-run' (Fig. 3) (Haynes et al. 2007; Wightman \& Haynes, 2004). During regulated exocytosis, vesicles storing transmitter molecules that are prepackaged for secretion actively move to specific sites at the plasma membrane (active zone) and fuse after stimulation by specific secretagogues. Fusion of vesicles with the plasma membrane has three consequences; the first is to incorporate integral membrane proteins, such as ion transporters or ion channels, etc. into the cell membrane. The second is to incorporate lipid material into the plasma membrane. The third is to release intra-vesicular contents into the extracellular space by exocytosis. In the first mode, referred to as 'full fusion' exocytosis, the fusion pore is thought to dilate, releasing the full vesicular contents outside the cell. The vesicle membrane is then assumed to completely incorporate into the cell plasma membrane and subsequently recycled through endocytosis with the endosomal compartment for repair and resorting. Full fusion has been described in neurons and endocrine cells (Fig. 3c, top) (Izquierdo et al. 2002). 

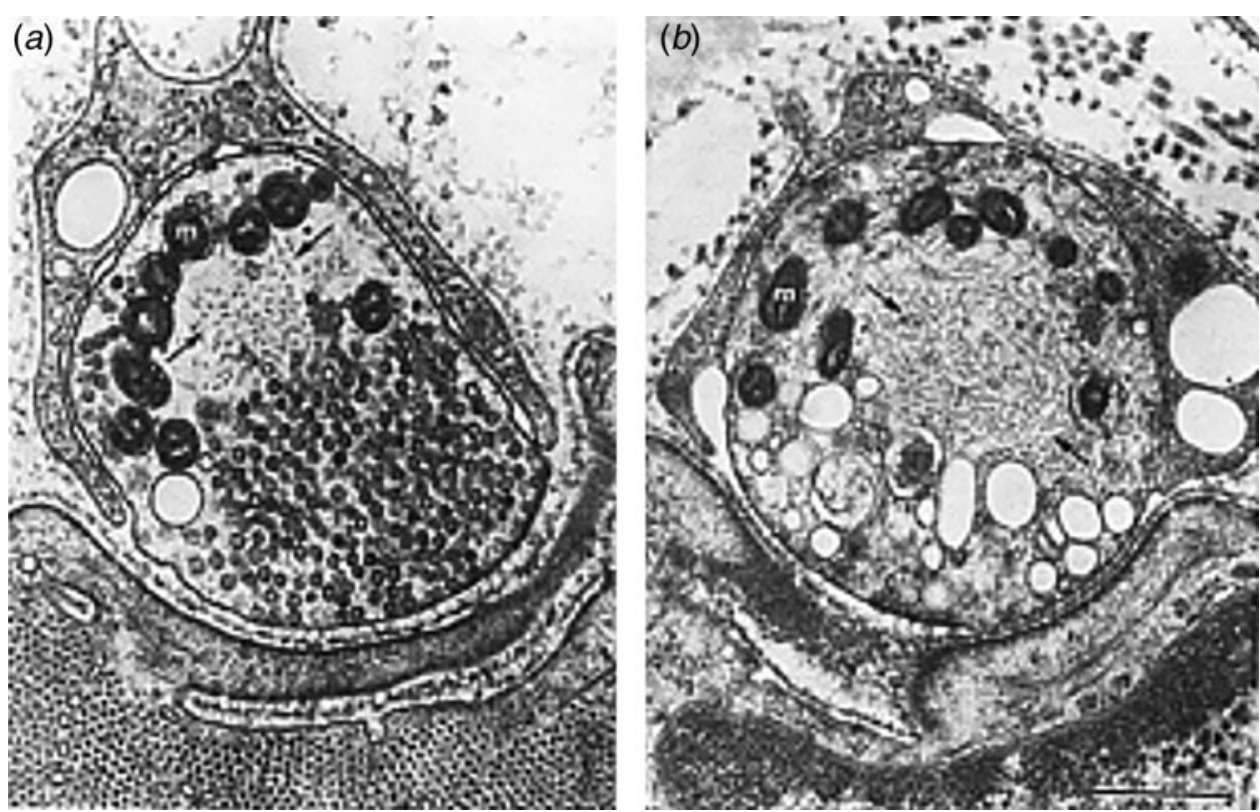

Fig. 2. $\mathrm{La}^{3+}$-induced depletion of synaptic vesicles and their replacement by large vacant endosomes during prolonged transmitter release at the frog neuromuscular junction (b), compared with a control nerve terminal (a) (Heuser \& Miledi, 1971). This morphological change was the first indication that synaptic vesicle membrane might recycle via standard mechanisms of endocytosis. Unlike other methods that existed for exhausting transmitter release, $\mathrm{La}^{3+}$ treatment did not appear to damage other components of the nerve including its mitochondria (m) or its core of neuro filaments (between arrows). Scale bar $=0.5 \mu \mathrm{m}$.

A large number of methods have been applied to measure the events during exocytosis, which have provided evidences for full fusion. The basics of techniques like amperometry, as well as fluorescence and patch clamp, are described in the next section. Amperometric measurements of release from catecholamine-containing vesicles and imaging of fluorescently tagged vesicle proteins and lipids reveal rapid release kinetics and have been assumed to measure full fusion events. Likewise, quantum dots with an $\sim 15 \mathrm{~nm}$ diameter that are preloaded in synaptic vesicles can be released, suggesting a fusion pore larger than $15 \mathrm{~nm}$ or vesicle collapse (Zhang et al. 2007).

The second mode, which is called the kiss-and-run mode, vesicles fuse with the plasma membrane by forming a transient fusion pore $(2-4 \mathrm{~nm})$ while preserving vesicle integrity, during which a small fraction of the vesicular contents is released. The pore then closes again and the vesicle is instantly recaptured. Data supporting the idea of transient fusion states were first established by patch-clamp techniques from the observation of capacitance flickers, during which a measurable conductance corresponding to a narrow pore of $0.5-3 \mathrm{~nm}$ diameter is sometimes detected in secretory cells, including nerve terminals (Lawson et al. 1977). By measuring capacitance and release via amperometric recordings, it has been shown that the capacitance flicker is sometimes accompanied by a smaller than normal amperometric signal in chromaffin cells and PC12 cells, suggesting that kiss-and-run may partially release transmitter. In recent years, morphological methods, such as fluorescent microscopy and atomic force microscopy (AFM) have provided evidence for the kiss-and-run model. Both morphological techniques showed that vesicles release their contents within seconds to minutes (Albillos et al. 1997). After the fusion event, the vesicles detach and return into the cytosol while maintaining their integrity and morphology.

Many studies have attempted to characterize the mechanism that triggers one or the other mode. Different domains of a synapse can control the choice between kiss-and-run and full fusion (Wang et al. 2003). It seems that some cells, such as chromaffin cells, can switch between kiss-and-run and full fusion by varying extracellular calcium. Physiological stimulation regulates the exocytotic mode through calcium activation of protein kinase $\mathrm{C}$ in chromaffin cells. At low $\mathrm{Ca}^{2+}$ concentrations, re-closure of the fusion pore is unlikely and vesicles preferentially incorporate completely into the plasma membrane, probably to be internalized later by a conventional mechanism that passes through endosomal structures. With increasing $\mathrm{Ca}^{2+}$ concentration, the probability and rate of re-closure of the fusion pore increases, leading preferentially to kiss-and-run events (Fig. 4) (Alés et al. 1999). Recently, observations from real-time recordings from dopaminergic neurons showed that dopamine from small synaptic vesicles was almost exclusively released from a flickering fusion pore, indicating that kiss-and-run is the predominant mode of exocytosis in these cells. Here we review the recent progress in understanding all modes of exocytosis and furthermore highlight the supporting evidence for another form of release that is apparently the dominant mode 
(a)
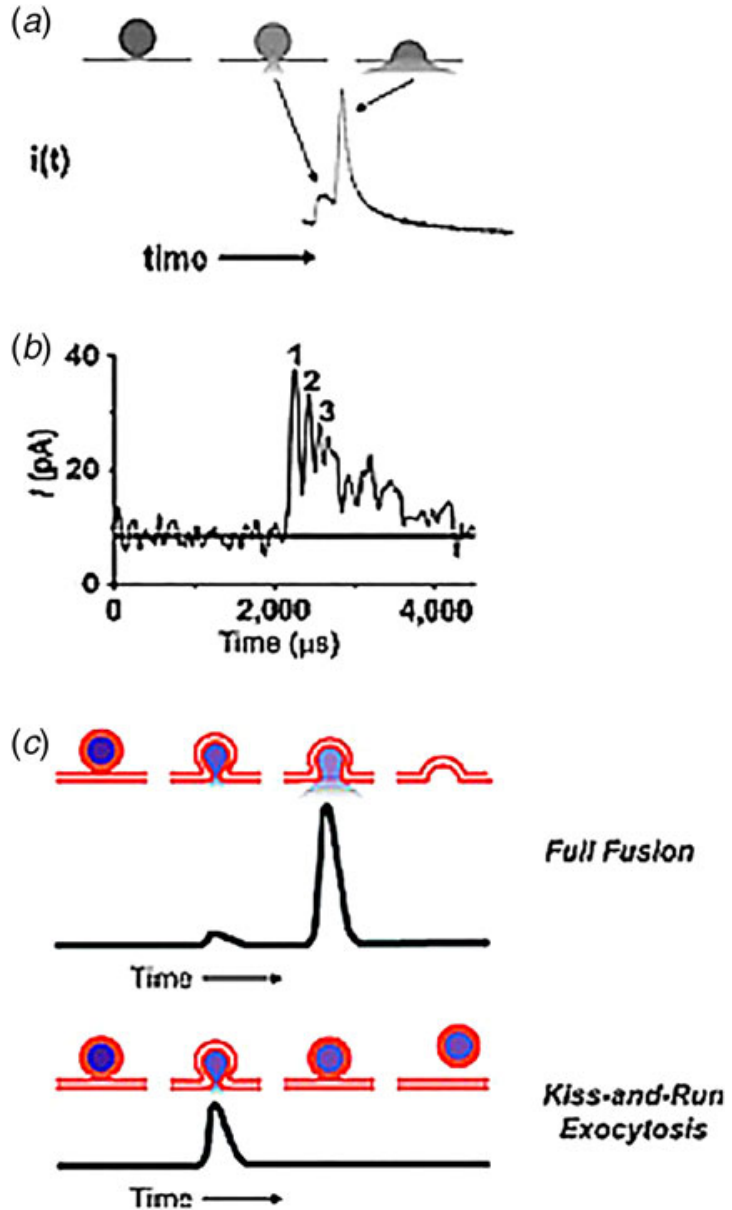

Fig. 3. Biophysical mechanisms of vesicular release can be elucidated from amperometric measurements at carbon-fiber microelectrodes. (a) Proposed mechanism and representative trace that depicts a prespike 'foot'. It is proposed that the small rise in current that precedes the distention of vesicular contents is due to formation of the fusion pore. This is followed by a large spike, which has been associated with full collapse of the vesicle membrane and expulsion of the transmitter contents (Haynes et al. 2007). (b) Amperometric trace that displays a 'flickering' fusion pore recorded from the stimulus-coupled secretion of dopaminergic mouse neurons. It is proposed that upon fusion with the plasma membrane, the fusion pore intermittently opens and closes as indicated by the complex peak observed. Each label on the complex event is assigned to a unique flickering event (Staal et al. 2004). (c) Cartoon depicting the mechanism of kiss-and-run exocytosis. This mechanism indicates partial release of a vesicle's content and can be resolved using electrochemical measurements as depicted in the simulated traces (Wightman \& Haynes, 2004).

during exocytosis. In this mode, which is distinguished from kiss-and-run exocytosis, we term open and closed exocytosis, the fusion pore appears to dilate further than 2-4 nm and more of its contents are transferred to the extracellular compartment. Then after signaling, the pore closes again preserving the vesicle.

\section{Techniques to monitor exocytosis}

The vesicle compartment encapsulates a variety of neurotransmitters e.g. dopamine, epinephrine, norepinephrine, serotonin, neuropeptides. Even though the concentration of neurotransmitter in a single vesicle is rather high, the small size of a single vesicle unit results in neurotransmitter release of the order of zepto- to femtomoles. These events are also very fast and occur on a sub-millisecond to millisecond time scale. Hence, to be able to study the exocytosis process, it is essential to use techniques with high sensitivity and high temporal resolution (Aravanis et al. 2003; Betz et al. 1996; Cahill et al. 1996; Jin et al. 2008; Lin et al. 2012; Omiatek et al. 2010; Pihel et al. 1994; Travis \& Wightman, 1998; Wang et al. 2014; Wightman et al. 1991; Xia et al. 2009). In exocytosis research, there has also been major development of new techniques that make it possible to probe the vesicle fusion and neurotransmitter release events, where most of the research has been focused on performance on live single secretory cell assays. This has made the study of exocytosis possible in detail with cell-to-cell variability. Methods to monitor the location, time course and to quantify individual exocytotic events have included 


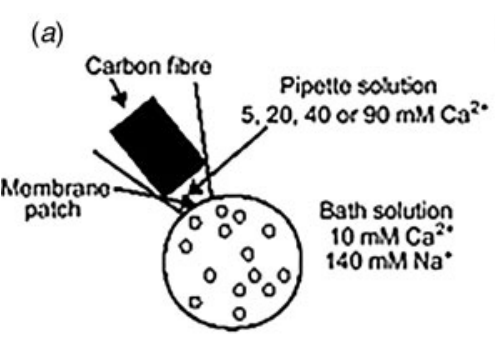

(b)

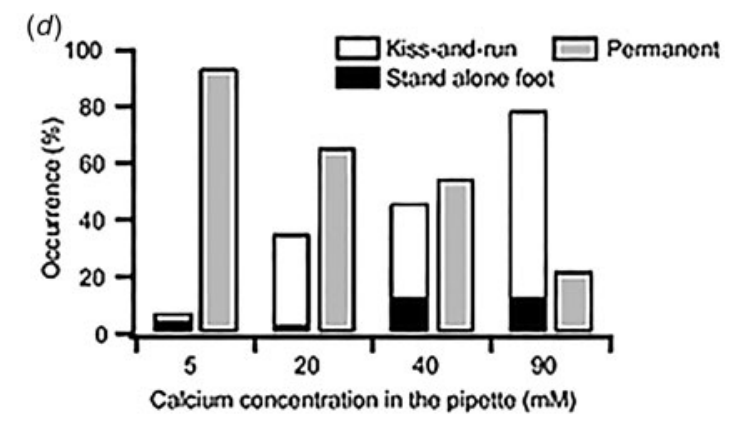

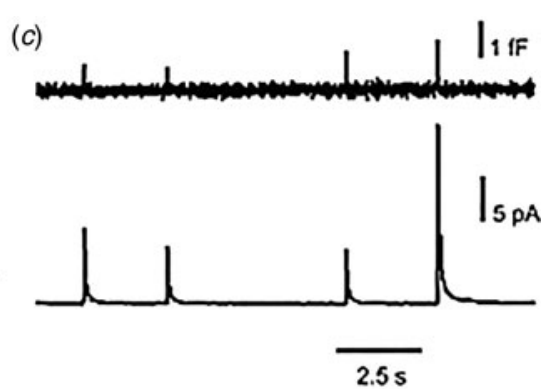

(e)

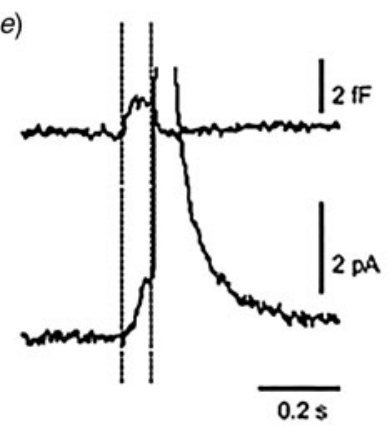

Fig. 4. Catecholamine release during fast kiss-and-run and permanent fusion events in rat chromaffin cells, recorded by patch amperometry. (a) The patch-amperometry technique. (b) Irreversible fusion events, recorded with $5 \mathrm{mM} \mathrm{Ca}^{2+}$ in the patch pipette. Top, capacitance trace; bottom, electrochemical detection of catecholamines by amperometry. (c) Fast kiss-and-run fusion events, recorded at $90 \mathrm{mM}$ $\mathrm{Ca}^{2+}$. (d) Percentages of transient fusion events and irreversible events depending on patch-pipette $\mathrm{Ca}^{2+}$ concentration. (e) Fast kiss-and-run event shown at an expanded timescale. Top, transient increase in capacitance; bottom, amperometric signal, showing a foot followed by an amperometric spike (Alés et al. 1999).

electrophysiology, electrochemistry, microscopy and spectroscopy. Critically, these methods measure different aspects of the vesicle from contents released to membrane associated with intravesicular dyes and membrane capacitance. The temporal resolution of these methods varies as well and we later postulate that a given technique might even be biased toward a specific kind of event. Here, we give an overview of the different methods in brief to clarify what each technique measures and the range of temporal resolution for each.

\subsection{Patch clamp}

In the 1970s, Neher and Sakmann developed the patch clamp technique for electrophysiological measurement of ion channel activity. By forming a seal of the cell membrane around the tip of a glass micropipette filled with electrolyte solution and containing an electrode, the changes in impedance of the cell plasma membrane can be recorded during ion channel activity due to the current flow through channels when opening (Neher et al. 1978). This technique was further modified for capacitance recordings of the surface membrane of adrenal chromaffin cells patched in whole cell mode and became an important tool in measuring exocytosis (Neher \& Marty, 1982). In the whole cell mode the membrane aspirated into the pipette is aspirated off creating an open connection to the cell cytoplasm. The capacitance of the cell plasma membrane is proportional to the membrane area. Discrete changes in capacitance are detected during exocytosis events due to vesicles fusing to the plasma membrane. The high sensitivity and the high temporal resolution of patch clamp capacitance technique allow resolution of the small changes in membrane area of a single vesicle compartment fusing and re-capturing at the plasma membrane.

\subsection{Amperometry}

Electrochemical techniques have been the dominant methods for quantifying chemical release during exocytosis. Single-cell carbon fiber amperometry has been one of the most widely used techniques owing to its high sensitivity and high temporal resolution (sub-millisecond) and was introduced in the 1990s by the Wightman lab to monitor chemical release from single vesicles at bovine adrenal chromaffin cells (Leszczyszyn et al. 1990; Wightman et al. 1991). Release of easily oxidized signaling molecules can be recorded and quantified by placing a carbon fiber microelectrode in close proximity to a cell. By applying a constant potential to the electrode, molecules secreted from a vesicle compartment against the electrode surface can be oxidized or reduced. This can be recorded as an oxidation or reduction current and provides information about the kinetics of 
individual vesicle release events. Hence, for each individual exocytosis event, a single current transient is observed. With Faraday's law $(Q=n N F)$, the charge $Q$ recorded from a current transient can be related to the number of moles of signaling molecules released from the vesicle, where $n$ is the number of electrons transferred in the reaction and $F$ is the Faraday's constant (96 $485 \mathrm{C} \mathrm{mol}^{-1}$ ). The shape of each current spike also provides kinetic information about the vesicle release by determining the current transient rise time (we typically use the time from 25 to $75 \%$ of the maximum of the ascending part of the spike) that is thought to correspond to the opening of the fusion pore, and the half width of the spike as measurement of the duration of the event. Additionally, the presence or absence of a pre-spike, often called a 'foot' that is indicative of the vesicle initial opening and the oxidation of molecules diffusing out of the initial pore, is considered to be important in exocytosis communication to discern information about kinetics and stability of the fusion pore opening and pore flickering before vesicle dilation (Amatore et al. 2007; Chow et al. 1992; De Toledo et al. 1993). Amperometry has also been used to identify different modes of partial vesicle release, such as fusion pore flickering (Bruns \& Jahn, 1995; Staal et al. 2004). Flickering fusion pores that open and close without further enlargement have been detected with carbon fiber amperometry and these kiss-and-run events have even been suggested to be the dominant mode of release during dopamine release at neurons. Carbon fiber amperometry has successfully been applied to a number of cell types including primary cultures, immortalized cell lines and nerve cells in vivo. Hence, amperometry is a very sensitive method with high temporal resolution that can quantify and resolve the kinetics of molecules released from single exocytosis events. However, amperometric detection of chemical release is limited to electroactive molecules including neurotransmitter molecules such as dopamine, serotonin, epinephrine and norepinephrine, and histamine. Therefore in more complex environments like the brain other electrochemical methods are needed (see cyclic voltammetry below).

\subsection{Patch amperometry}

In whole cell mode, patch clamp capacitance has good temporal resolution to measure the net effect of single vesicle fusion and fission over the whole cell membrane, but does not monitor what comes out of the vesicle. Patch amperometry was introduced by Lindau and coworkers in 1997 by combining the patch clamp capacitance technique with amperometry (Albillos et al. 1997). This was achieved by inserting a carbon fiber electrode inside the tip of a patch clamp pipette. The change in capacitance from the increase in membrane from secretory vesicles fusing to the plasma membrane during exocytosis is measured simultaneously with amperometric measurement of release. From recordings of patch amperometry in whole cell mode it was for instance shown that the concentration of neurotransmitter content, based on amperometric determination of amount molecules released from a vesicle, does not change with vesicle size and they suggested that elevated concentrations of extracellular calcium shift the mode of exocytosis from what was apparently full exocytosis to kiss-and-run mode (Alés et al. 1999). This technique has also been performed on cell-free inside-out membrane patches from adrenal chromaffin cells to probe the intracellular environment upon fusion pore formation and endocytosis (Ales et al. 1999). Chow et al. combined patch clamp capacitance measurements on bovine adrenal chromaffin cells in whole cell mode together with amperometry detection by placing a carbon fiber electrode in close proximity to the cell (Chow et al. 1992). These experiments showed that when cells were stimulated to exocytosis an increase in capacitance and an oxidation current from release of catecholamines were simultaneously detected. It was noted that from many of the current spikes, a foot, was detected and thought to correspond to neurotransmitter molecules leaking through a stabilized initially formed fusion pore before fusion pore dilation and full vesicle content release. Similar experiments were performed at beige mouse mast cells and showed that often a flickering behavior of the fusion pore is observed before the bulk of vesicular release. This is detected by a flicker in capacitance and confirmed with simultaneous amperometric recording showing that leakage of histamine and serotonin occur through this flickering fusion pore and is observed as a pre-spike foot before pore dilation and a subsequent larger transient current corresponding to a quantal release from the vesicle (Dernick et al. 2005).

\subsection{Cyclic voltammetry}

An electrochemical technique that can both quantify and offers selectivity is cyclic voltammetry. In this technique the potential at the electrode is linearly swept as a triangular wave. This potential sweep starts from a potential where the analyte is not oxidized and is scanned until reaching a potential where the analyte monitored is oxidized in a diffusion-limited manner. This is followed by linearly reducing the potential until the oxidized species is reduced back again at the electrode surface, which is detected as a reduction current. This results in a cyclic voltammogram that gives characteristic oxidation and reduction peaks that can serve as a fingerprint to identify an analyte. To optimize the temporal resolution to the time needed for exocytosis measurements a fast scan cyclic voltammetry method has been developed. Here the triangular wave is performed and followed with a resting period at the initial scanning potential that is about ten times longer than the scan time. This is set to the time it takes to re-generate a new layer of analyte at the surface of the electrode. Fast scan cyclic voltammetry is particularly useful because the voltammogram provides information to identify the detected substance. However, the response time, selectivity, 
and sensitivity depend on the surface state of the electrode (Ciolkowski et al. 1992; Pihel et al. 1994). With cyclic voltammetry, Wightman and coworkers were able to identify catecholamines secreted from bovine chromaffin cells (Leszczyszyn et al. 1990; Wightman et al. 1991). Selectivity by cyclic voltammetric measurements has also been used to distinguish release of epinephrine and norepinephrine at bovine adrenal medullary cells (Ciolkowski et al. 1992; Pihel et al. 1994).

\subsection{Fluorescence microscopy imaging}

Whereas electrochemical methods can monitor the dynamic processes of fusion pore formation and quantify neurochemical release, optical methods can be used to observe the vesicle transport inside the cell. Hence, the vesicle granule movement in the cell and its path before docking and fusion with the plasma membrane can be studied. Fluorescence microscopy imaging of exocytosis has been credited to the use of fluorescence markers that can be integrated with the vesicle membrane, conjugated to synaptic membrane proteins, or encapsulated within the synaptic vesicle unit. The styryl dyes, FM1-43 and FM4-64, are some of the most commonly used probes to stain the vesicle membrane. These hydrophilic molecules also have a hydrophobic part to their structure and therefore are easily incorporated to the leaflet of a lipid bilayer. Hence, when cells are stimulated to exocytosis in the presence of the dye, the dye can be encapsulated into the vesicle compartment when the fusion pore opens or during subsequent endocytosis following full exocytosis. The partitioning of the dye into the inner leaflet of the vesicle membrane increases the quantum yield of the dye significantly compared with that in solution, which is beneficial in eliminating the background in bulk solution and allowing the stained vesicle compartments to be visualized as bright spots inside the cell. When the cell is stimulated to exocytosis, the dye is unloaded from the vesicle membrane and is monitored as a decrease in fluorescence signal (Betz et al. 1996; Zenisek et al. 2000). Styryl dye labeling of the vesicle membrane has been readily used to study the kinetics of exocytosis and the kinetics of vesicle cycling. Styryl dye labeling of cells, in combination with encapsulation of a single quantum dot inside the vesicle, has also been used to determine the mode of exocytosis in cells (Betz \& Bewick, 1992; Betz et al. 1992; Zhang et al. 2009b). As the efficiency of loading vesicles with styryl dyes is so high, and if kiss-and-run is rare, then it should be a logical conclusion that vesicles are actually undergoing mostly an open and closed form of exocytosis to maintain this loading efficiency.

To selectively illuminate a subpopulation of the vesicles in the cell, the fraction of the cell near the membrane can be illuminated using total internal reflection fluorescence microscopy (TIRFM) (Akopova et al. 2011). In TIRFM an excitation light beam traveling in a solid (e.g., a glass coverslip or tissue culture plastic) is incident at a high angle upon the solid/liquid surface to which the cells adhere. That angle, measured from the normal, must be large enough for the beam to totally internally reflect rather than refract through the interface, a condition that occurs above some 'critical angle'. Total internal reflection generates a very thin (generally less than $200 \mathrm{~nm}$ ) electromagnetic field in the liquid with the same frequency as the incident light and exponentially decaying in intensity with distance from the surface. This field is called the 'evanescent wave' and is capable of exciting fluorophores in the liquid near the surface while avoiding excitation of a possibly much larger number of fluorophores farther out in the liquid (Axelrod, 1989). In a typical TIRFM setup, evanescent waves that are generated by totally reflected incident light at a glass-water interface penetrate into an adherent cell on the glass surface with a depth less than $200 \mathrm{~nm}$. Only the features and events at the plasma membrane and the cytoplasmic region close to the plasma membrane are illuminated and visualized using the evanescent field. In this way, TIRFM eliminates potential fluorescence background from much of the cytosolic region that may obscure the events close to the cell surface (Wang et al. 2008). Using this technique, Steyer et al. stained single vesicles with acridine orange, a $\mathrm{pH}$-sensitive dye and showed that vesicles approaching close enough to the plasma membrane were illuminated and could be tracked during the transport in the cytoplasm prior to and during docking and fusing with the plasma membrane (Steyer et al. 1997). During exocytosis, the fluorescence brightness from these vesicles is observed to diminish as the fluorescent probe escapes out of the vesicle compartment. TIRFM imaging has been used to study vesicle paths and location before and during vesicle fusion and to probe fusion pore dynamics and mode of exocytosis. Akopova et al. examined ATP secretion from A549 cells by TIRFM to directly visualize ATP-loaded vesicles and their fusion with the plasma membrane (Akopova et al. 2011). A549 cells were labeled with quinacrine or Bodipy-ATP, fluorescent markers of intracellular ATP storage sites and time-lapse imaging of vesicles present in the evanescent field was undertaken. Under basal conditions, individual vesicles showed occasional quasi-instantaneous loss of fluorescence, as expected from spontaneous vesicle fusion with the plasma membrane and dispersal of its fluorescent cargo. Hypo-osmotic stress stimulation (osmolality reduction from 316 to $160 \mathrm{mOsm}$ ) resulted in a transient, several-fold increment of exocytotic event frequency. Lowering the temperature from 37 to $20^{\circ} \mathrm{C}$ dramatically diminished the fraction of vesicles that underwent exocytosis during the 2-min stimulation, from $\sim 40$ to $\leqslant 1 \%$, respectively, although this was not observed for exocytosis in platelets (Ge et al. 2009). Parallel ATP efflux experiments with a luciferase bioluminescence assay revealed that pharmacological interference with vesicular transport (brefeldin, monensin), or disruption of the cytoskeleton (nocodazole, cytochalasin), significantly suppressed ATP release (by up to $\sim 80 \%$ ), whereas it was completely blocked by N-ethylmaleimide. Chapman and coworkers have used TIRFM with a pHluorin to examine exocytosis from PC12 cells 
and concluded that the longer events taking more than $2 \mathrm{~s}$ were full release and the events shorter than $2 \mathrm{~s}$ were from kissand-run exocytosis (Zhang et al. 2011). Although a powerful method to visualize vesicles movement and fusion, fluorescence imaging has two disadvantages. One is that it cannot count the molecules released as can be done with amperometry. The second is that most experiments to date have been temporally limited to the longer realm of 100s of microseconds to seconds.

\section{Traditional models of exocytosis}

Until the last decade, exocytosis has generally been thought to be carried out via a full distention of the vesicle membrane into the plasma membrane. The early electron microscopy experiments, patch clamp and fluorescence all led to models where the bulk release process was all or none. Ultrastructural studies for example show that omega structures exist, but the existence of these structures does not necessarily prove that full fusion is the major pathway. Later techniques followed the exocytotic process dynamically, allowing exocytosis to be monitored in terms of single events. In general these techniques either monitor the content that is released or the fate of the vesicle membrane during and following the event. In this section we extract the evidence for full distention from several of these studies, which use chromaffin cells as a model for exocytotic release.

One early advance in the dynamic tracking of exocytotic events was the application of patch clamp to monitor changes in membrane capacitance during exocytosis (Neher \& Marty, 1982). By applying a sine wave in voltage clamp configuration Neher and Marty were able to measure a signal that was roughly proportional to membrane capacitance. A lock-in amplifier was used to extract the phase corresponding to the membrane capacitance. Assuming that the plasma membrane approximates a capacitor and that a change in capacitance is directly related to an increase in the surface area of the membrane, the fusion of a single vesicle with the cell membrane can be monitored. The amplitude of the signal tracked membrane fusion and fission events in real time. This experiment showed that the increase and subsequent decrease in membrane surface area associated with exocytosis stimulation in chromaffin cells occurred in a stepwise manner indicating that full distention events were the major exocytosis events. Later, capacitance measurements done in mast cells identified three types of events categorized as on steps, off steps, or flickers (Fernandez et al. 1984). The flickers were taken as the immediate retrieval of the vesicle material from the plasma membrane thus providing early evidence for kiss-and-run exocytosis. The majority of events recorded were irreversible, thus the flicker event was assumed to be a specialization of the fusion event as opposed to the primary mode of release. Still later, a study showed that under certain conditions, $90 \%$ of the release events measured at calf adrenal chromaffin cells were in fact transient (Elhamdani et al. 2006).

Patch clamp results show that exocytosis is carried out by at least two fusion mechanisms; one in which the vesicle membrane fuses irreversibly to the plasma membrane, which is called full fusion and one where the membrane fusion is transient. The fusion events measured, however, might not account for all the release events expected from stimulation of exocytosis. One indication of this assertion is that the number of events measured at chromaffin cells in patch clamp experiments appears to be lower than the events measured with amerometry experiments. While 50 events can be measured from one stimulation (Wightman et al. 1991) at a chomaffin cell using amperometric probes, the patch clamp experiments mentioned here report 2.5 and 7 events per cell, respectively. As a first approximation the difference in number of events is striking. A second observation to be made is the duration of the kiss-and-run events presented. The duration of these events presented by later work are in the fastest case an average of $100 \mathrm{~ms}$ and in early work they can last several seconds. This time scale is consistent with the flickers reported by Fernandez et al. (1984), which appear to have durations greater than 500 ms. Again looking to amperometry, the majority of events measured at these cells are less than 100 ms, usually significantly less, producing a disconnect, which must be rectified to understand the nature of these measurements.

A second way to track membrane dynamics as they occur during exocytosis was developed in total internal fluorescence microscopy, which only excites fluorescence a few $100 \mathrm{~nm}$ into the sample, thus only interrogating the membrane and the immediately adjacent cytosol. Furthermore, because the evanescent wave used for excitation decays exponentially, the intensity can be used to approximate a depth scale. By labeling the membrane of secretory granules in chromaffin cells with VAMP-GFP, the diffusion of the label into the plasma membrane was used to hypothesize that approximately $90 \%$ of the events measured correspond to full distention (Allersma et al. 2004). Comparing the numbers of events measured once again to amperomery gives similar results to those, which were found in patch clamp. The cited work reports 139 events measured from the surface of the cell contacting the glass slide after 3 stimulations and a second reports 147 events (Schmoranzer et al. 2000). These numbers also appear quite low when compared with the number of events measured with amperometry. Likewise the duration of the detected events is extended as compared with amperometric measurements. Distention of the vesicle takes on the order of a full second according to TIRFM measurements, whereas the release measured with amperometry is typically completed in a less than $100 \mathrm{~ms}$. This discrepancy makes it difficult to ensure that the membrane sensitive techniques are in fact measuring the same phenomenon that the content-sensitive amperometry measures. 
While it is difficult to directly implicate full distention as the major mode of content release, the fact that full distention occurs is overwhelmingly supported by the results from both the patch clamp and TIRFM experiments. These experiments show that material from the vesicle membrane is transferred to the cell membrane, either through the diffusion of a label or through the increase in membrane capacitance. Fluorescence and capacitance measurements have been combined in chromaffin cells using the styryl dye FM1-43 as a marker for the surface area of the of the cell and it was found that increases in capacitance are paired with incorporation of the labeled membrane into the cell membrane (Smith \& Betz, 1996). However it has also been shown that in some cases capacitance changes are not coupled to surface area changes (Kilic, 2002). In this work the author argues that the surface area remains constant because the addition of membrane relieves tension in the membrane. They furthermore suggest that the FM1-43 signal cannot be detected in the membrane because it is sensitive to changes in tension, supporting the idea of incorporation of new membrane without a subsequent increase in membrane surface area. In this way, it can be hypothesized that vesicle material is released via a full distention mechanism of exocytosis. These experiments, however, do not track content release and thus might exclude events that release through a more transient membrane interaction and likewise might register membrane events that do not correspond to content release. Indeed, tracking content release is critical for the understanding of these mechanisms.

Amperometry allows one to basically count and track molecules released during exocytosis. The advantage of amperometry is its high temporal resolution and coulometric efficiency, which ensures that the full amount of released molecules from nearly every event under the electrode will be measured. Thus it is not surprising, as was mentioned above, that this technique records large numbers of fast exocytotic release events. The quantal nature of the spikes might be indicative of full release in that the size of the vesicle would be a straightforward mechanism for maintaining quanta. Later work showed that current transients could also appear as so called stand-alone feet, which might be an indication of a second release modality. Often, an amperometic spike will have a pre-spike feature, which appears as a small rise in current. This feature, again called the foot of the spike, is interpreted as the diffusion of a small amount of catecholamine through an initial stable fusion pore. The standalone foot then is interpreted as a pore, which releases content and either closes vis-à-vis kiss-and-run, or fully distends after the vesicle is empty. These stand-alone foot events account for about $20 \%$ of the events measured from bovine chromaffin cells and release on average $77 \%$ of the amount of catecholamine as compared with the foot on normal amperometric spikes (Zhou et al. 1996). Additionally the events are significantly longer than the average amperometric peak, lasting as long as $250-300$ $\mathrm{ms}$ in some cases, corresponding better to the events detected using TIRFM. However, monitoring exocytosis with electrochemistry only allows one to monitor release with no way to monitor the fate of the secretory vesicle. It seems clear that there is at least a possibility that this disconnect between the time scales of events and numbers of events measured between the two techniques might indicate two processes, a slow one that is coupled to membrane distension and a fast one that is involved in content release.

Understanding the relationship between the membrane fusion event and the content release event requires simultaneous monitoring of both processes. This has been achieved in several laboratories by using combinations of the techniques discussed above. In one study content release of fluorescently labeled neuropeptide Y was monitored using TIRFM, while membrane fusion was monitored using whole cell capacitance measurements (Becherer et al. 2007). The question probed here was whether the events measured at the bottom of the cell (TIRFM) were representative of the events measured over the whole cell (capacitance). The conclusion was that the events were in fact comparable, demonstrating that irreversible membrane events are coupled to peptide release in the cell. The number of events recorded were, as previously shown from these techniques, comparatively low when compared with amperometry, with the TIRFM measurement recording about 4 events per cell on the bottom and capacitance recorded approximately 40 events over the whole cell suggesting the these techniques, while they appear to be sensitive, might not detect all forms of content release, i.e. the modes that are detected with amperometry. An earlier work coupled capacitance measurements to confocal imaging of neuropeptide release from two neuroendocrine cell lines (INS-1 and PC12) (Barg et al. 2002). They showed that peptide release lagged the membrane fusion and initial pore formation by $1-10 \mathrm{~s}$. This delay is attributed to the size of the peptides, which excludes them from transiting through the pore. They suggested that it is not until the membrane fully distends that the peptides are released. These studies indicate that the slow release events measured with fluorescent techniques are full fusion events and that capacitance measurements apparently favor these irreversible and relatively slow events.

Amperometry has been coupled to whole cell capacitance measurements and in general the integral of the current measured is coupled to an irreversible increase in membrane capacitance (Chow et al. 1996; Vonruden \& Neher, 1993). One interesting observation made with this experiment was that rapid stimulation in the form of photolysis of caged calcium led to a lag between capacitance increase and content release. Several possibilities have been proposed including a calcium induced decrease in quiescent endocytosis, the possibility that secretion may be taking place at 'hot spots' at some distance from the electrode, or that the initial capacitance signal is related to the fusion of empty vesicles. At any rate these studies show 
that the capacitance signal is difficult to interpret without some additional characterization. A more sophisticated version of this experiment was subsequently carried out on chromaffin cells by using patch amperometry (Albillos et al. 1997). In this technique, true simultaneous measurements of the same membrane area are made. The data presented showed that the capacitance steps that correspond to amperometric spikes are predominantly irreversible indicating full membrane distension is the dominant mode of release. Furthermore, kiss-and-run events were identified to account for $10 \%$ of the measured events. The amperometric signal from these events indicated that the vesicle was completely evacuated before closing and that the release lasted on the order of seconds. This experiment suggests that the fast event is coupled to distention and the slow event is coupled to immediate endocytosis further complicating the view of this process.

The initial conclusion to be drawn from these experiments is that distention events and release events are generally coupled. However, a closer look reveals that the amount of material released from individual vesicles measured with patch amperometry is generally considerably larger than the amount measured using standard amperometry. A quick survey of the charges measured at chromaffin cells using amperometry gives values of $\approx 1 \mathrm{pC}$ (Wightman et al. 1991), 0.67 pC (Montesinos et al. 2008) and 0.62 pC (Segovia et al. 2010), and charges measured using patch amperometry are 2.2 pC (Albillos et al. 1997), 1.8 pC (Montesinos et al. 2008) and 3.2 pC (Segovia et al. 2010). This suggests that each of these methods cater to a specific event, but it is difficult to make direct comparisons. However, there are at least two experiments in the literature that carry both methods, one shows that the average charge measured using patch amperometry is nearly triple the values measured using amperometry (Montesinos et al. 2008) and a second shows that the charge increases to more than five times when using patch amperometry (Segovia et al. 2010). It appears that the patch influences the size of the quanta released. If we can make the assumption that the patch neither affects the size of the granule nor the concentration of the catecholamine with in it, then it follows that the charge measured using amperometry corresponds to less than the total content of the vesicle. Thus, the typical amperometric spike represents a partial content release while the patch extracts all or at least more of the content. This concept will be discussed in further detail in the section dealing with variations in amount of chemical release.

The work discussed here probably raises more questions than it answers, because the results depend largely on the method of measurement. Patch clamp and TIRFM show that full distention definitely occurs; however, determining whether it is the primary form of release is challenging because it is difficult to monitor content release and especially partial release with both these methods. Amperometry tracks content release with high temporal resolution and apparently detects relatively more events when compared with the membrane sensitive techniques. The implication here is that the membrane-based techniques might miss many release events. The events measured with amperometry also tend to be faster suggesting the possibility that release is partial with occasional slow events detected. The slower events might be related to the delayed release of content measured with TIRFM. Here, the catecholamine might be partially secreted through a small fusion pore during an initial opening, recorded as a stand-alone foot in amperometry. Following the lag, full distention could occur, which would appear as a second long event in TIRFM. The relatively low occurrence of long events in amperomety measurements might indicate that this event is rare and that TIRFM is not sensitive to the major content release events measured with normal shorter events in amperometry. Patch amperometry shows that spikes are coupled to irreversible capacitance steps; however, it also shows that the average event measured using amperometry does not correspond to a full release of vesicle content. This result suggests but does not prove that the major content release measured using amperometry infact a partial release. It seems possible that the events measured using patch clamp methods have been influenced by the patch configuration, by either facilitation of full release, perhaps via membrane tension, or by increasing the efficiency of the amperometric measurement.

\section{Evidence where partial release during exocytosis appears to be dominant \\ 4.1 The total content of a secretory vesicle is greater than the amount released}

The apparent content of individual vesicles containing electroactive neurotransmitters such as dopamine has been inferred through measurements of release by electrochemical methods such as amperometry. In 1998, Pothos et al. reported quantal release from dopamine neurons by placing a carbon fiber electrode on the axonal varicosities and detecting the release of dopamine electrochemically after release was stimulated with $\mathrm{K}^{+}$or $\alpha$-latrotoxin (Pothos et al. 1998). Results indicated that an average of 3000 molecules were released over a $200 \mu$ s event and that the quanta released per event could be modulated with pharmacological drugs known to effect neurotransmitter synthesis and increased expression of protein transporters, which accumulate them (Pothos et al. 2000). While these results indicated the amount of dopamine released through exocytosis, the total vesicle content might not be the same. How much neurotransmitter is compartmentalized into an individual secretory vesicle? When two techniques are used to study the same question, new and interesting results can be found. In 2008, Borges and coworkers used both conventional amperometry as well as patch amperometry at a single cell where suction is applied to a part of the membrane in order to create a seal and allow for capacitance measurements to study wild-type and 
mutant chromaffin cells (Montesinos et al. 2008). Although not the focus of the study, comparison of the average quanta released per vesicle by these two approaches provides interesting information about the nature of each technique. With conventional amperometry, the total change per event was $0.67 \pm 0.08 \mathrm{pC}$, whereas patch amperometry revealed that the total charge to be $1.81 \pm 0.09 \mathrm{pC}$ for the same cell line. The greater release detected through patch amperometry is presumably due to the applied suction on the membrane, which might make complete release more likely, indicating that what is commonly seen with conventional amperometry might not be full fusion, but be partial release. A partial release of $40 \%$ can be inferred by comparing the conventional and patch amperometry data discussed above.

In 2009, Ewing and coworkers developed a method to determine the total vesicle content by studying vesicles in vitro (Omiatek et al. 2009). In this method they coupled capillary electrophoresis to electrochemistry to first separate isolated vesicles from PC12 cells, and then detect their contents amperometrically by allowing them to lyse on the surface of a carbon fiber electrode through a method called electrochemical cytometry (Fig. 5). The Ewing group then went on to compare measurements of release with conventional amerometry to this new cytometry technique for the same line of cells, allowing them to quantify what is released during an individual exocytosis event, using amperometry, and the total contents of an individual vesicle, using cytometry (Omiatek et al. 2010). Using PC12 cells, Omiatek et al. estimated that only around $40 \%$ of the total dopamine content seems to be released during a single exocytosis event. Thus, the remaining $60 \%$ of the vesicular contents were not released. Significantly less catecholamine was measured by stimulated exocytosis at single cells, $141 \pm 3$, versus $387 \pm$ $2 \mathrm{zmol}$ for isolated vesicles measured on the microfluidic platform. Partial exocytosis appears to be highly likely. Comparison of release events and vesicle content by these methods is shown in Fig. 6. Omiatek et al. also ably examined the effect of pharmacological drugs known to effect dopamine release from cells, specifically L-DOPA, which accumulates dopamine, and reserpine, which decreases dopamine content. In these studies it was determined that total vesicle content is altered dramatically by addition of these agents, but that the vesicles continued to release roughly $40 \%$ of their total dopamine content.

In another report, electrochemical cytometry was used to study the mechanisms of vesicular content modulations in vivo (Omiatek et al. 2013). By dosing live animal models and studying the resulting vesicular content, it was demonstrated that medications such as reserpine and L-DOPA alter the synaptic vesicle transmitter content in rats in a time dependent manner. Additionally, and in agreement with the previous study, this work showed that vesicular dopamine content was higher than expected. More recently, individual vesicle content was quantified with a new approach. Here a $33 \mu \mathrm{m}$ electrode is placed in a suspension of secretory vesicles isolated from chromaffin cells and then the electrochemical response of the vesicles is measured as they rupture. The impact events occur stochastically allowing for quantification of individual vesicles (Fig. 7) (Dunevall et al. 2015). The vesicles appear to adsorb onto the electrode surface and sequentially spread out over the electrode surface, trapping their contents against the electrode. These contents are then oxidized, and a current (or amperometric) peak results from each vesicle that bursts. A large number of current transients associated with rupture of single vesicles (86\%) are observed under the experimental conditions used, allowing them to quantify the vesicular catecholamine content. Although preliminary, if one compares the measured average number of catecholamines per vesicle $\left(4.31 \times 10^{6}\right.$ molecules $)$ to published results for amperometrically measured release from adrenal cell vesicles, the amount released during exocytosis is in the range of $40-60 \%$ of the amount contained in the average vesicle. It should be noted that although this is similar to other measurements, there are issues with signal to noise differences between the methods that need to be taken into account before numbers like this can be certain. However, this indeed suggests that the average vesicle contains considerably more catecholamine than observed in a single exocytotic release event, which provides more evidence for partial release in an open and closed form of exocytosis.

In a further investigation, the Ewing group used flame-etched carbon-fiber microelectrodes to obtain conical nanotips and used these for intracellular detection of the catecholamine content of individual nanoscale vesicles in live PC12 cells (Li et al. 2015). The nanotip conical carbon-fiber microelectrodes were pushed through the cell membrane without significant damage. In this way, the active conical electrode surface was exposed to catecholamine-containing vesicles in the cell interior (Fig. 8) (Li et al. 2015). The total vesicular catecholamine content measured by intracellular vesicle electrochemical cytometry (114500 \pm 15300 molecules) is significantly different from the amount detected for stimulated exocytosis in single-cell amperometry experiments with the same electrodes (73 $200 \pm 5820$ molecules). According to these data, it appears that approximately $64 \%$ of the total catecholamine content of the vesicles is released during stimulated exocytosis in these PC12 cells. Although the fraction release is somewhat higher than measured previously for PC12 cells, this result again supports the hypothesis that exocytosis is characterized by an open and closed mechanism.

\subsection{The majority of exocytotic events are followed by immediate endocytosis}

Using Quartz Crystal Microbalance with Dissipation (QCM-D), Cans et al. were able to study both exocytosis and endocytosis simultaneously for a population of cells (Cans et al. 2001). In the QCM-D measurement, two secreting cell types, PC12 and 
(a)

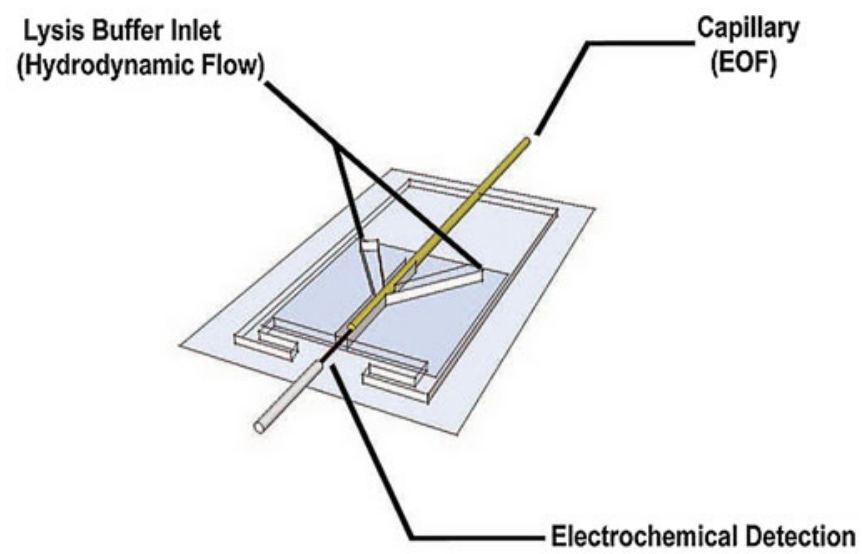

(b)
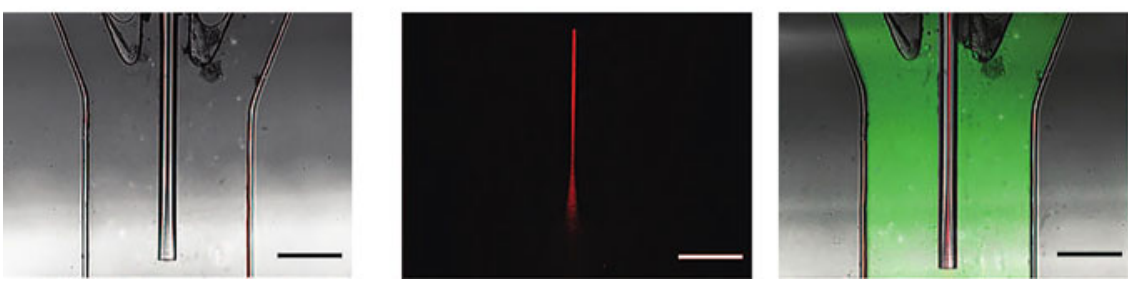

Fig. 5. Electrochemical cytometry with a three layer PDMS device for the end-column lysis and electrochemical detection of vesicles separated by capillary electrophoresis (Omiatek et al. 2009). (a) Schematic of device (not drawn to scale). (b) Bright field and confocal fluorescence images of the device.

NG 108-15, were grown to confluence on a quartz crystal coated with oxidized polystyrene. Exocytosis was stimulated by introduction of high $\mathrm{K}^{+}$.

Solution and changes in frequency and dissipation were monitored simultaneously. For both cell types, results showed that the release from the cells could be detected by a shift in frequency after stimulation (loss of mass). The mass lost was completely regained in a matter of seconds (Fig. 9) (Cans et al. 2001). To determine where exocytosis and endocytosis began and ended, the data were fit to a three-state model consisting of three consecutive first order irreversible reactions in which the first state (the original state) represents the release of mass by the cells, i.e. exocytosis, and the second state is followed by recovery and uptake of mass, i.e. endocytosis, and finally, a slower phase where the frequency descends lower than the original baseline and might result as the cell structure recovers from release. The results of the model provided time constants for each of the three phases as well as the amplitude of the frequency change when one phase ended and the next began. By comparing the predicted frequency to the experimentally determined frequency they were able to determine that exocytosis and endocytosis significantly overlap. With careful consideration of their data it appears more than $97 \%$ of exocytosis events undergo immediate endocytosis due to the differences in the magnitude of the theoretical and experimental frequency maxima between the first two states. In these experiments the dissipation measurement is correlated to the rigidity of the cells. Although three phases were apparent for frequency measurements, only two are apparent for dissipation measurements and relate to first an increase in rigidity of the cells, followed by a recovery period after which the cells are more elastic. Again, these results indicate the retrieval of material after exocytosis that allows the cells to return nearly to their initial state.

\subsection{Full distention of the vesicle may not be necessary for quantal release}

In 2010, Amatore et al. studied the maximum aperture angle necessary for a vesicle to completely release its contents based on a physiochemical simulation of exocytosis and compared with several individual event spikes recorded by amperometry (Amatore et al. 2010). The model was based on a vesicle fusing to the cell membrane and the consequences of constraining the membrane during release of vesicular contents, based on the radius and the angle of aperture for the vesicle. They determined that the vesicle only needs to open to about $10^{\circ}$ in order to release its contents and provide the transient signal observed, as opposed to completely fusing with the cell membrane and fully dilating (Fig. 10) (Amatore et al. 2010). This indicates that at the end of a release event the vesicle matrix is still mostly covered by membrane, and not exposed to the extracellular environment, suggesting that full fusion is unlikely for these events. 


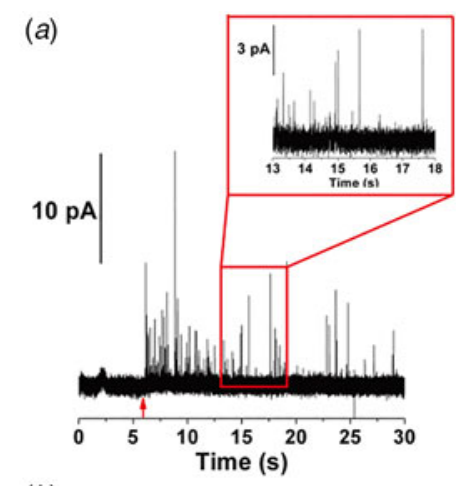

(b)

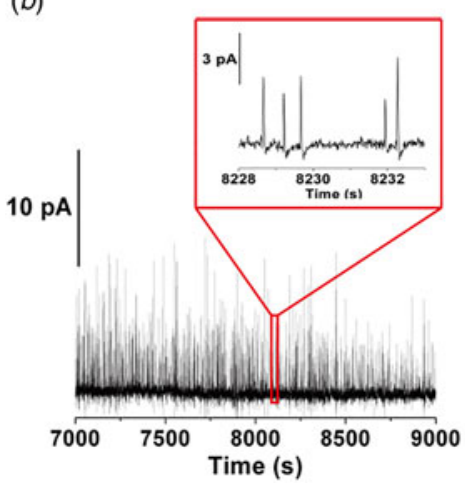

$(c)$

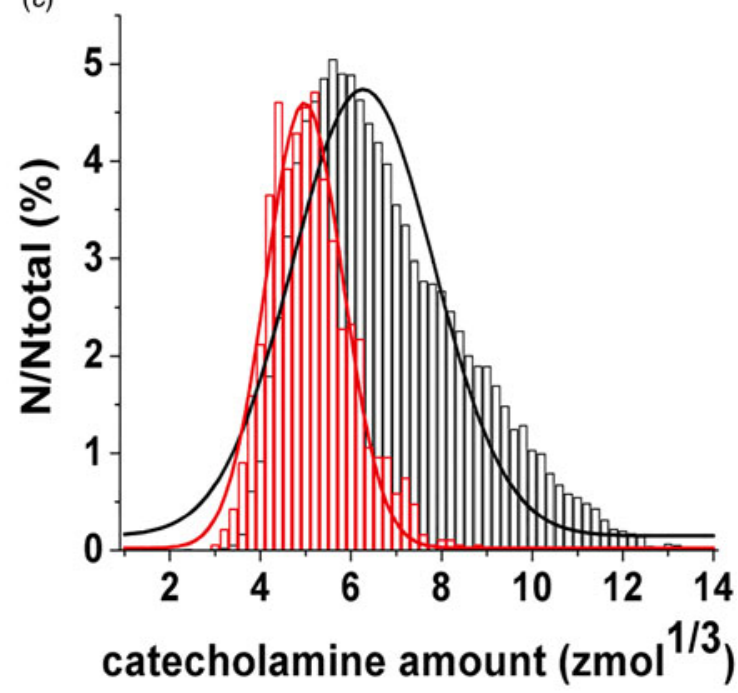

Fig. 6. Amperometric quantification of catecholamine amounts released from during exocytosis in PC12 cells with amperometry and in vesicles with electrochemical cytometry (Omiatek et al. 2010). (a) Representative amperometric trace resulting from exocytotic release at intact cells. Red arrow indicates where an elevated $\mathrm{K}^{+}$application was used to induce exocytosis. (b) Electrochemical cytometry of individual vesicles. $(c)$ Normalized frequency histogram of the vesicular catecholamine amounts quantified from intact cells that underwent stimulated exocytosis (red) versus isolated vesicles (black) investigated with electrochemical cytometry.
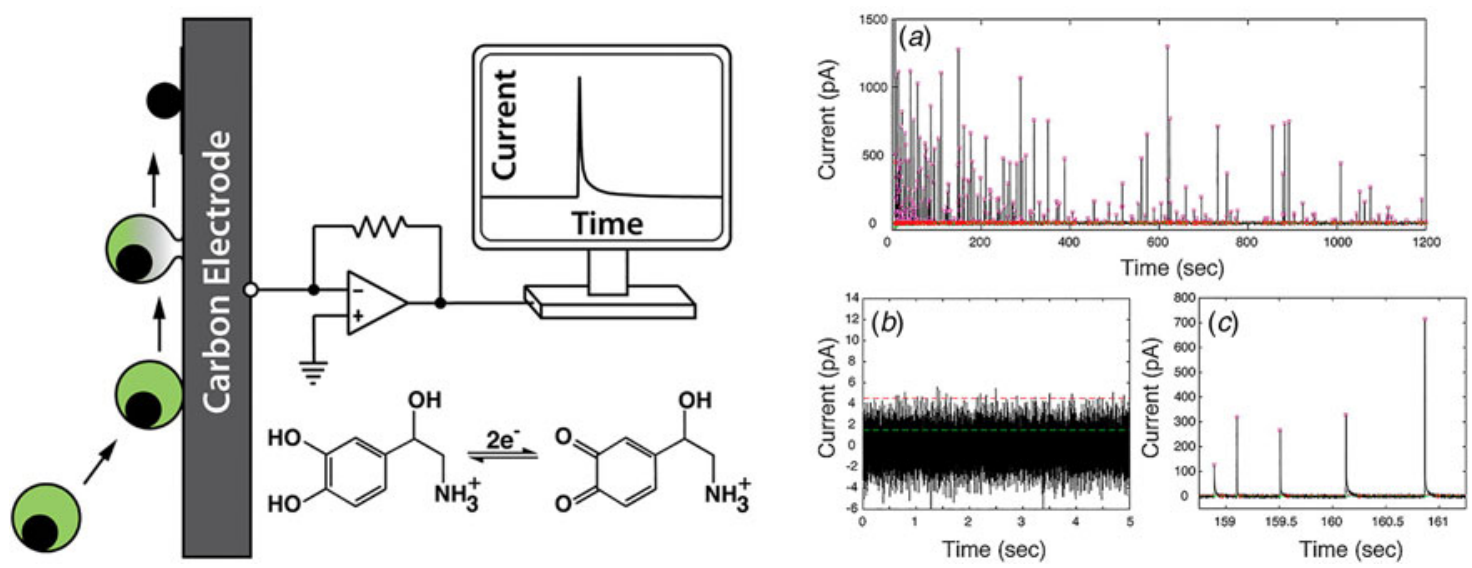

Fig. 7. The electrochemical response to single adrenal chromaffin vesicles filled with catecholamine hormones as they are adsorbed and rupture on a $33 \mu \mathrm{m}$ diameter disk-shaped carbon electrode. (a) Representative trace from a suspension of chromaffin cell vesicles. (b) A 5 -s baseline at $0 \mathrm{mV}$ versus $\mathrm{Ag} \mid \mathrm{AgCl}$ in the presence of vesicles. (c) Expanded view of current transients. The pink squares represent the $I_{\max }$ of all peak candidate peaks submitted for further analysis. The green line represents the root-mean-square (RMS) and the red line five times the RMS of the baseline noise (Dunevall et al. 2015).

Previous work by Frolov et al. focused on the point connecting the vesicle to the plasma membrane, called the neck (Frolov et al. 2003). Here model systems of the neck were created using a patch pipette and a stable Teflon ring. When a lipid film is attached to both points the stability and shape of the neck connecting the two can be studied as a function of the length of the neck. Their model demonstrates that this neck structure can have more than one stable stationary shape, which are dictated by 
(a)

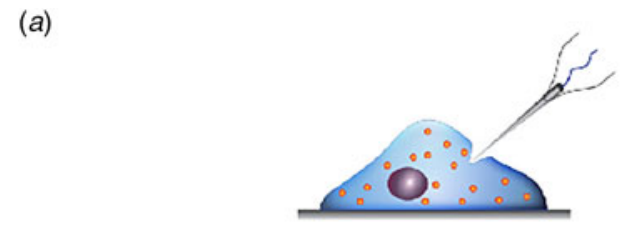

สิ
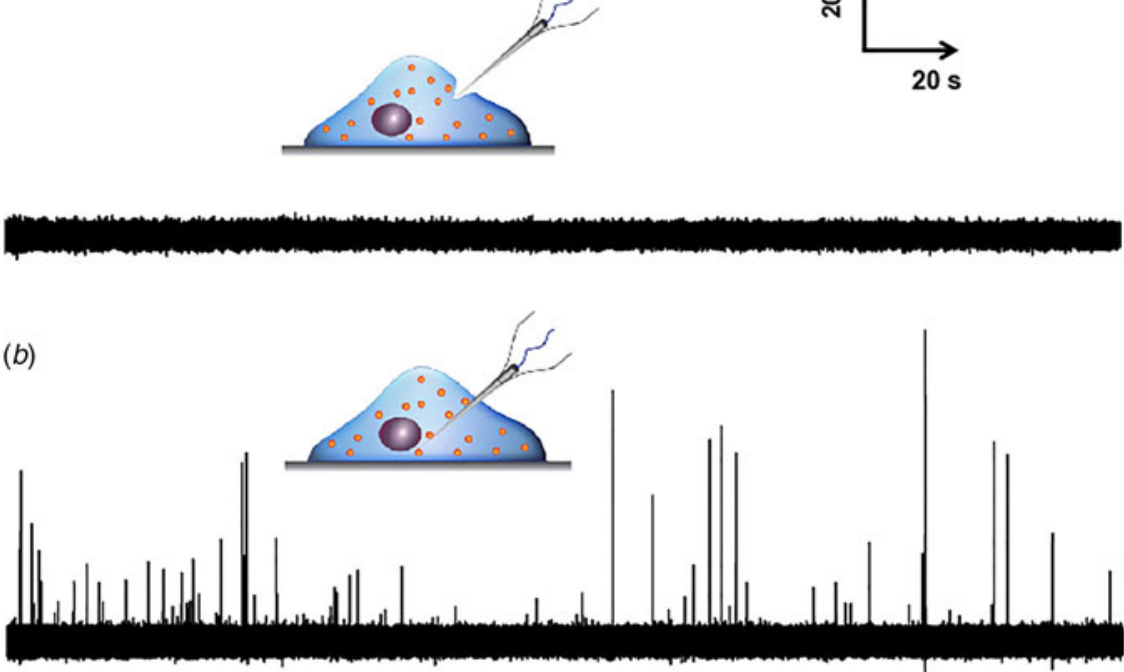

$(c)$

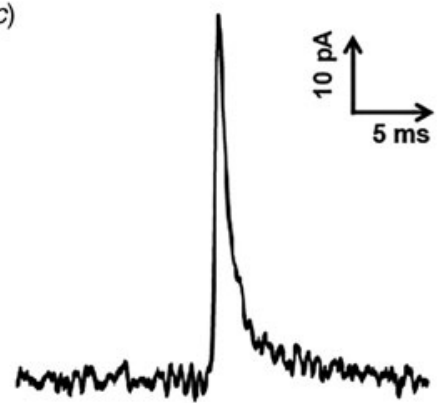

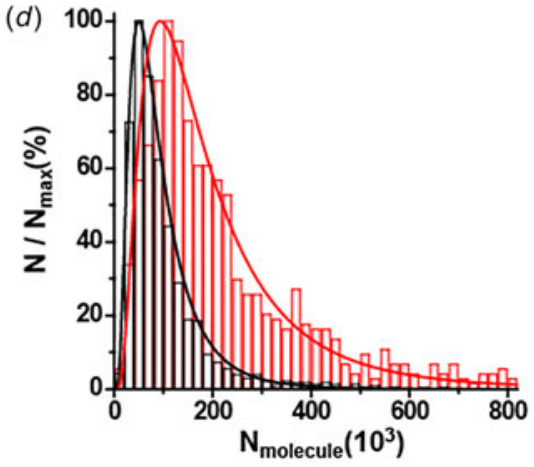

Fig. 8. Intracellular electrochemical vesicle impact cytometry (Li et al. 2015). (a,b) Amperometric traces for a nanotip conical carbonfiber microelectrode pushed against a PC12 cell without breaking into the cytoplasm (a) or placed inside a PC12 cell (b). (c) Amplified amperometric current trace. $(d)$ Normalized frequency histograms describing the distribution of the vesicular catecholamine amount as quantified from untreated PC12 cells by intracellular vesicle electrochemical cytometry (red, $n=1017$ events from 17 cells) and by $\mathrm{K}^{+}$-stimulated exocytosis at the same electrode (black, $n=1128$ events from 17 cells). Bin size: $2 \times 10^{4}$ molecules. Fits were obtained from a $\log$ normal distribution of the data.

the lateral tension and bending rigidity of the system. This work further suggests a mechanism for providing a transient opening of the neck, similar to what may be present during 'kiss-and-run' exocytosis, where the permeability of the neck can be 'toggled' by the different stable states, and might even 'flicker'. This concept might play an important physiological role, maintaining the compartments where two environments meet (Frolov et al. 2003).

\subsection{A flickering fusion pore might regulate quantal release and vesicle recycling}

The concept of 'flickering' has been studied in two ways, both of which relate to the regulation of release from a synaptic vesicle. First, it has been used to define the variations or fluctuations in an open fusion pore (amperometry), and secondly it has been used to define the opening and closing of an individual fusion pore (patch amperometry). In a conventional amperometric trace, a single rise and decay are linked to the release of material from a synaptic vesicle. Staal et al. published data indicating that a portion of these events actually consists of more complex events. In $25-30 \%$ of the events, the pore appears to open and close more than once, each opening expelling small quanta of neurotransmitter into the synaptic cleft. The evidence for this can be found in the analysis of the individual event. Each complex event has 'flickers' in which a distinct increase and decrease in current can be detected above the background noise level. These flickers are part of the larger peak considered to be the release from a single vesicle, where the total quanta released and duration $\left(t_{1 / 2}\right)$ are greater for complex events. With each flicker a decrease in the amount of dopamine released is detected (Staal et al. 2004). They further found that the incidence of complex events, but not the number of events per flicker, could be regulated pharmacologically. When neurons were 

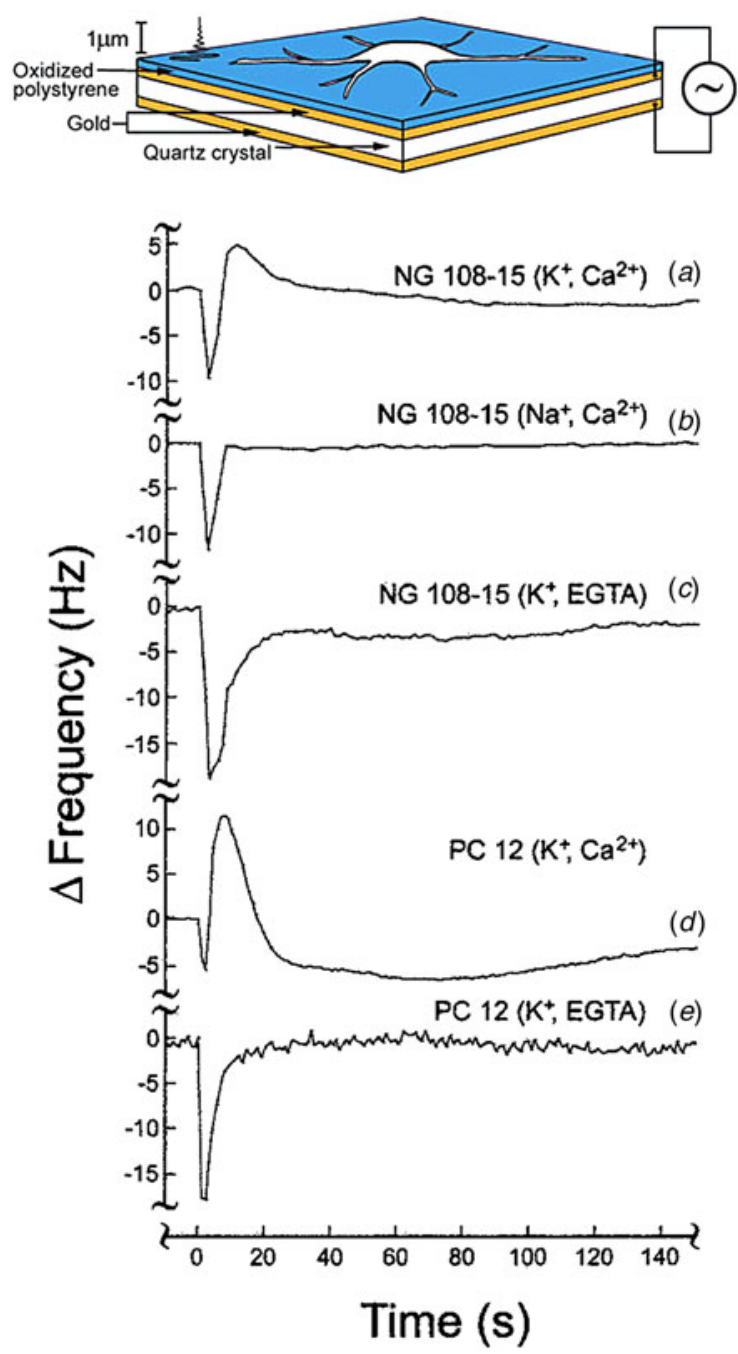

Fig. 9. Schematic representation showing a cell grown on an oxidized polystyrene-coated piezoelectric quartz crystal and outlining the layered configuration of the device (Cans et al. 2001). Detection of frequency changes in populations of cells following high potassium stimulation. Changes in frequency $(\Delta f)$ were recorded with the QCM-D for $(a)$ NG 108-15 cells grown on polystyrene-coated quartz crystals that were exposed to high- $\mathrm{K}^{+}$media, $(b)$ a control experiment in which the NG 108-15 cells were rinsed with physiological buffer only, $(c)$ a control experiment in which NG 108-15 cells were depolarized in the presence of 3 mM EGTA, $(d)$ PC 12 cells exposed to high-K media and, $(e)$ a control experiment in which PC 12 cells were stimulated in the presence of $3 \mathrm{mM}$ EGTA. The stimulation buffer was $90 \mathrm{mM} \mathrm{K}^{+}$for all stimulations with $2 \mathrm{mM} \mathrm{Ca}^{2+}$ added $(a, b, d)$.

treated with phorbol 12,13-dibutyrate (PDBU), which increases the size of the readily releasable pool of vesicle, the incidence of complex events decreases. In contrast, staurosporine was found to nearly double the occurrence of complex events. These data have implications for both vesicle recycling and dopamine signaling. The rapid release and closure of vesicles through this flicker process might allow the vesicle to release small amounts of material without losing its entire contents, thus skipping the longer process of vesicle recycling through clathrin-coated pits, and allowing the vesicle to be involved in more signaling events. Similarly, the ability to release small amounts of neurotransmitter might modulate how far a signal travels. Models of dopamine diffusion in social synapses suggest that larger quanta can travel farther and have the ability to stimulate more neurons in the network, while smaller quanta may not be able to reach as far and only stimulate neurons in closer proximity.

In patch amperometry, the release events of most patch amperometry events are thought to represent full collapse of the synaptic vesicle and incorporation of the new membrane material into the plasma membrane. This is usually seen by an up-step, or increase in capacitance across the membrane derived from the increase in total surface area under study. According to the Wu group at the National Institute of Neurological Disorders and Stroke in the USA, only $80 \%$ of events are from full release 


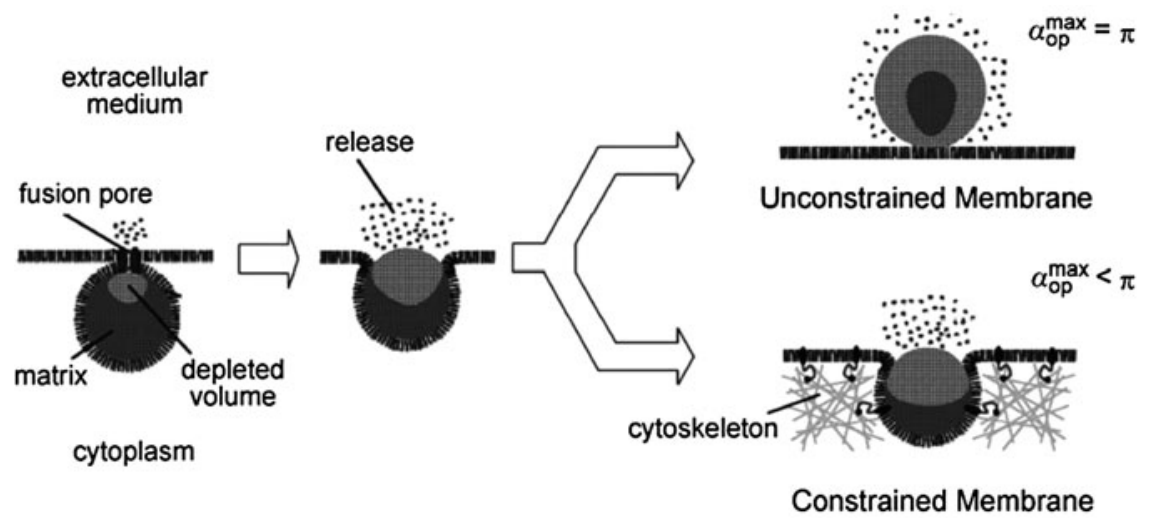

Fig. 10. Schematic membrane showing how the structural relationship of the vesicle with the membrane cytoskeleton network may constrain the maximum aperture angle (Amatore et al. 2010).

events causing the collapse of the vesicle, even though suction is applied directly to the membrane during study. The remaining $20 \%$ of events are regarded as 'flickers' in which an up-step in the capacitance, and therefore in membrane area can be detected, followed by a down-step back to baseline after a $10 \mathrm{~ms}-1 \mathrm{~s}$ period. In all cases these steps are low frequency $(0.088$ $\mathrm{Hz}$ ) indicating that it is a single vesicle fusion, stimulated by $\mathrm{K}^{+}$addition and not random events, and of a size predictable by electron microscopy (He et al. 2006). These two modes can be related back to full fusion where the collapse of the vesicle indicates all content has been expelled, and open and closed exocytosis where the flickers indicate a quantal release from a vesicle that may then be recycled back into the cell, and might control the quantal size and amplitude.

\subsection{Nanometer-sized pores can be seen as 'feet' associated with amperometric spikes}

First reported in 1992 by Chow et al. a foot is a low current feature associated with an amperometric spike found on a portion of release events (Chow et al. 1992). These features can be described by the formation of a stable pore, longer than $1 \mathrm{~ms}$ (typically tens of ms) with an amplitude $3 \times$ the noise, where small amounts of neurotransmitter are released in addition to the spike feature detected with all exocytosis events. Feet have been detected in the three forms; a ramp is a linear increase in current until spike onset, while a plateau is a stabilized current preceding the spike, which can have a fast or progressive onset.

The first reports by the Neher group of pre-spike feet introduced a new concept to the molecular mechanisms of exocytosis. Further measurements using patch amperometry showed that a small fusion pore, less than $3 \mathrm{~nm}$, is present and stable before it expands during exocytosis. Additionally they suggested that some of the pores that are transient might be capable of releasing the entire contents of the vesicle without ever fully distending (Albillos et al. 1997). With PC12 cells, pharmacological treatments with reserpine (known to decrease catecholamine content) and L-DOPA (known to increase catecholamine content) were found to modulate the charge and duration of pre-spike feet (Sombers et al. 2004). Careful analysis of these pre-spike events in chromaffin cells has revealed that they are quite common, occurring in approximately $30 \%$ of events, and that although their occurrence does not indicate the size of the event, the flux through the corresponding fusion pore correlates with the flux of the entire event (Amatore et al. 2009). Feet have been detected both before (Amatore et al. 2005) and after (Mellander et al. 2012) the spike for a significant number of events leading to a higher total release from the vesicle as measured by the charge released.

Features detected at the termination of exocytosis are called post-spike feet. These feet are harder to detect due to the decay of the spike at the end of exocytosis, but Mellander et al. were able to identify stable plateau features occurring at the end of exocytosis, with a portion of events having both pre and post spike feet. They analyzed amperometric peaks corresponding to release at PC12 cells and find stable plateau currents during the decay of peaks, indicating closing of the vesicle after incomplete release of its content (Fig. 11) (Mellander et al. 2012). These post spike features have lower current than pre-spike features, but a similar total charge was released that indicates the closing of the vesicle after a partial release of contents. Taken together the presence of pre-spike and post spike features demonstrates that a stable fusion pore exists not only before, but also after the release event that has been previously thought to be complete exocytosis. This evidence indicates that at least some so-called full release events involve first the formation of a fusion pore, through which some content is released, followed by a dilation of this pore, where the typical release event occurs, and then ending in the narrowing of the pore and reduction in the flux of contents, and finally closing (Mellander et al. 2012). It was also observed that with amperometry, about $2 \%$ of all events are of a larger category releasing more than 200000 molecules from the average event at a PC12 cell (it is important to 
(a)

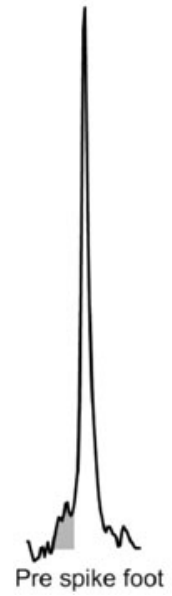

市|
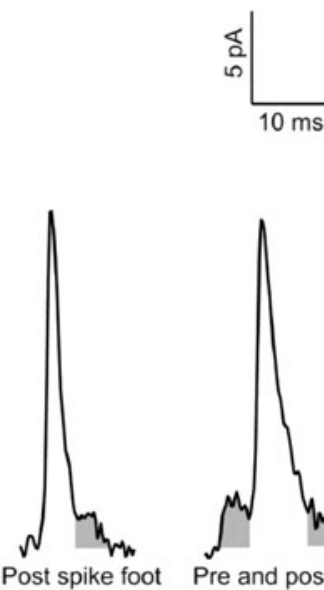

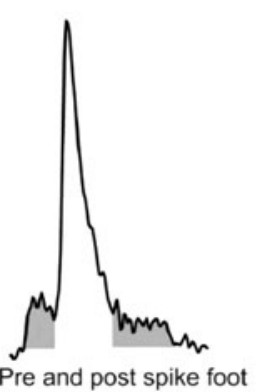

(b)

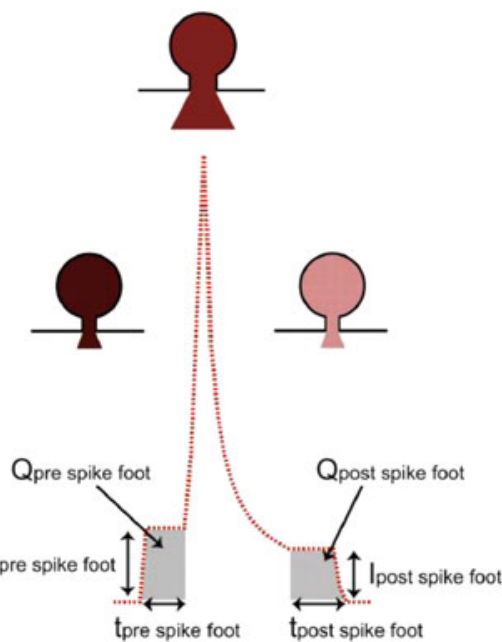

Fig. 11. (a) Three example peaks from PC12 cells. The first peak displays the traditional plateau foot, here termed pre-spike foot. The second peak shows what is referred to as a post spike foot, while the third peak displays both types of feet. $(b)$ Definition of the foot features that were used in the analysis. The lifetime of the foot $\left(t_{\text {foot }}\right)$, the current of the foot $\left(I_{\text {foot }}\right)$ and the charge of the foot $\left(Q_{\text {foot }}\right)$ for both pre and post spike feet (Mellander et al. 2012).

note that most spike analysis software programs eliminate these slow, larger events from consideration in data analysis). Finally, if one assumes that the foot height is related to concentration in the vesicle and that during the larger 'full' release stage much of the content is released, then the post foot relative to the pre foot should reflect the fraction released. This was examined for exocytosis where both pre and post feet were observed and the percentage released appeared to be about $40 \%$.

\section{Factors affecting the extent of partial release during individual events}

We argue that the average exocytosis event from dense core vesicles represents partial release of the neurotransmitter content. This hypothesis provides a rationale for the numerous observations of variations in the fraction of transmitter released to be manipulated and represents a new pathway for secreting cells to regulate the amount of transmitter released without a mechanism involving up or down regulating the amount of transmitter inside the vesicles. We have found evidence for possible mechanisms of this regulation in the literature, and also evidence that the method used to study exocytosis can affect the fraction released.

\section{1 Lipid composition of the plasma membrane}

The most direct evidence that the fraction released can be altered was presented by both the Amatore and Ewing groups who showed that the amount of neurotransmitter released per event can be quickly altered by influencing the lipid composition of the plasma membrane (Amatore et al. 2006; Mellander et al. 2012; Uchiyama et al. 2007). In one case, they found that a shortterm incubation of PC12 cells with the inverted cone shaped lipid lysophosphatidylcholine (LPC) increased the average amount of dopamine released per exocytosis event while incubation with the cone shaped phosphatidylethanolamine (PE) decreased it. In the other case, a rapid alteration of amount released per event was demonstrated by Amatore and coworkers who used LPC and arachidonic acid (AA), which displays a cone shape similar to that of PE, to study the effect of membrane lipid composition on the dynamics of exocytosis from bovine chromaffin cells. They found that LPC facilitates catecholamine release (rate, event frequency, charge released) while AA inhibits the exocytotic processes. They also observed that the detected amount of neurotransmitters in the presence of LPC was larger than under control conditions, while the opposite trend is observed with AA (Amatore et al. 2006).

Mellander et al. further presented direct evidence for a closing fusion pore by demonstrating that in addition to feet on the ascending part of the spike, some peaks display feet of the descending part of the spike. Furthermore, the ratio between post spike foot current and pre-spike foot current was found to decrease following PE incubation and increase following LPC incubation, strengthening the hypothesis of alterations of the fraction released (Mellander et al. 2012). A 48-h incubation with phosphatidylcholine (PC) has been shown to decrease the amount of dopamine released per event from PC12 cells while increasing the vesicular size. However, since the incubation scheme in this study was so long it cannot be excluded that the 
treatment had some effect on the dopamine concentration in the vesicles (Uchiyama et al. 2007). Depletion of cholesterol from PC12 cell membranes has also been shown to decrease the average of the quanta released, an effect that was abolished through replenishment of membrane cholesterol (Zhang et al. 2009a).

High curvature lipids can influence the properties of the membrane by adding positive or negative intrinsic curvature. The curvature is, however, also tightly coupled to the bending rigidity of the membrane. It has been speculated that the intrinsic curvature of the membrane is only significant at structures of nanometric dimensions, which is the size scale that we are looking at in the case of vesicle fusion. The first stage of vesicle fusion is the formation of a nanometer sized lipidic pore connecting the inside of the vesicle to the outside of the cell. Some neurotransmitter can escape through this pore. If the pore is stable for long enough, this leakage gives rise to the foot discussed above in some detail, preceding the peak resulting from the expansion of the pore. The magnitude of the foot current depends on the concentration of neurotransmitter inside the vesicle as well as the dimensions of the fusion pore. Amatore et al. have shown that incubation with LPC increases the pre-spike foot current. This was explained by the added positive curvature by addition of LPC to the outer leaflet of the cell, forming the inner leaflet of the fusion pore, which was hypothesized to result in a pore of a larger diameter. Likewise, addition of the negative curvature lipid, AA, resulted in a decrease in foot current, indicating a smaller fusion pore diameter (Amatore et al. 2006). Further supporting the hypothesis of membrane regulation of exocytosis, studies have shown that lipid incubation can manipulate the rate of decay of amperometric peaks, indicating that the vesicle is in fact closing again following partial release of its contents since the membrane composition should not influence a decay based on diffusion and consumption of the electroactive neurotransmitter at the electrode alone (Uchiyama et al. 2007). The results reported here together indicate that altering the lipid composition of the plasma membrane influences the lipid structures formed during exocytosis and that these structures can potentially govern the amount of neurotransmitter that escapes the vesicle during fusion.

\subsection{Mechanically applied pressure to the membrane}

The effects of membrane lipid composition on the kinetics and efficiency of release suggest that membrane dynamics seem to play a part in the regulation of transmitter release. When studying exocytosis with patch amperometry a pressure is applied to the patched membrane. Reviewing the literature, as mentioned above it seems like this pressure causes the vesicles to release a larger fraction of their neurotransmitter content as a result. Borges and coworkers studied release from wild type and chromogranin A deficient mouse chromaffin cells using patch amperometry configuration and amperometry of un-patched cells (Montesinos et al. 2008). When studying release from cells in the patch amperometry configuration, the charge released was almost tripled in both cases. De Toledo and coworkers similarly used amperometry and patch amperometry to study the influence of synaptotagmin-7 on exocytotic release from chromaffin cells (Segovia et al. 2010). The amperometry results indicate an average release of $0.62 \mathrm{pC}$ from wild type cells while the patch amperometry result shown an average amount of $3.24 \mathrm{pC}$ for the same cells, again suggesting that the full amount of neurotransmitter inside the vesicle is not expelled when the cell membrane is not under tension. The increase in released catecholamine from vesicles where the cell membrane is under tension from the patch pipette could be explained by the full collapse of the vesicle into the plasma membrane and the compete release of neurotransmitters. It could also result from a delay in vesicle fission following partial release, allowing the vesicle to release a larger fraction of, or even its entire, content. Another possibility is that the actin sub-membrane cytoskeleton and its linked proteins are dissociated from the releasing vesicle and can no longer control the fusion pore expansion and closing stages.

\subsection{Osmolality}

The osmolality of the solution surrounding the secreting cell is known to play an important role during exocytotic release. The concentration of catecholamine (epinephrine and norepinephrine) in bovine chromaffin cell vesicles is estimated at about 550 $\mathrm{mM}$ with additional ions and molecules resulting in a total concentration exceeding $750 \mathrm{mM}$. This effective osmolarity is lowered by association and binding of molecules to each other resulting in a vesicle that is isotonic with $300 \mathrm{mM}$ solutions (Holz, 1986). Altering the osmotic environment of the cell has been demonstrated to both induce and inhibit exocytotic release, indicating a vital role in this process. High osmolality conditions have been shown to inhibit exocytosis in a number of different cell types (Brown et al. 1978; Englert \& Perlman, 1981; Hampton \& Holz, 1983; Pollard et al. 1977). This inhibition appears to occur at a stage subsequent to the entry of $\mathrm{Ca}^{2+}$ into the cell (Holz, 1986). The induction of exocytosis in low osmolality solutions was first hypothesized to stem from a stress in the vesicle membrane caused by swelling of the vesicle, allowing the vesicle membrane to fuse with the plasma membrane, whereas the effect of a hyperosmotic solution was suggested to be based on the lack of swelling of the vesicles in this system, inhibiting fusion (Finkelstein et al. 1986; Holz, 1986). It has, however, since been shown that fusion in mast cells precedes vesicle swelling indicating that fusion is not the critical step in the osmotic regulation of exocytosis (Zimmerberg et al. 1987). Wightman and coworkers studied exocytotic release from chromaffin cells 
using carbon fiber amperometry and found that under hyperosmotic conditions, no major release events were detected; however, they did observe stand-alone feet. These results are in agreement with those from the Zimmerberg group (Zimmerberg et al. 1987) indicating that release is inhibited at a stage later than fusion. A factor hypothesized to be affected by the osmolality during release is the dense core vesicle protein matrix. Chromaffin cell secretory granules and dense core vesicles are packed with chromogranins that make up a large part of the vesicle matrix in addition to messenger molecules. These acidic proteins help lower the osmotic pressure of the vesicle by complexing with the catacholamine cargo. The important role of chromogranins for the vesicular storage capacity has been demonstrated by Borges and coworkers who showed that chromaffin cell vesicles from Chromogranin A deficient mice display a catecholamine vesicle content reduced by $30 \%$ compared with wild type mice (Borges et al. 1997; Montesinos et al. 2008). Hyper- and hypo-osmotic solutions have been shown to decrease the total amount of catecholamine released from a population of chromaffin cells without significantly influencing the influx of $\mathrm{Ca}^{2+}$ (Hampton \& Holz, 1983). This result could be explained with a decrease in release frequency or a decrease in released amount per vesicle, or both. Wightman and coworkers studied the effect of altering the osmotic pressure on individual cells using carbon fiber amperometry, allowing for a direct measure of quantal size and release frequency from bovine adrenal medullary cells. They found that moderately increased osmotic pressure $(630 \mathrm{mosm})$ caused a decrease in the amount of neurotransmitter released per fusion event and a decrease in release frequency. When the osmolarity was increased even further (970 mosm), exocytotic release was diminished to stand alone feet, as mentioned previously. The decrease in release amount and frequency was found to be unrelated to calcium influx as this was unaltered as monitored by imaging with the calcium sensitive dye Fura-2. After stimulation with barium under 970 mosm, flushing the cell with isotonic saline induced release (Borges et al. 1997). In a separate study, Wightman and coworkers measured release from cells in hypoosmotic buffer, which resulted in an increase in charge released from $1.7 \mathrm{pC}$ during control conditions to $2.9 \mathrm{pC}$ in hypoosmotic conditions. When the cells were returned to isosmotic buffer the amount released was again decreased to $2 \cdot 1 \mathrm{pC}$ suggesting that the full vesicular content is not expelled under the control isotonic conditions (Schroeder et al. 1996). The mechanism for this alteration of quantal release is probably the effect of a change in the rate of dissociation of transmitter from the matrix, possibly in combination with an altered fusion pore expansion rate. These findings suggest that the osmotic imbalance between the vesicle and surrounding media is important for regulating the number of molecules released from the vesicle during fusion. This mechanism requires that release is partial.

\subsection{Temperature}

By studying amperometric recordings of release from mast and chromaffin cells, Pihel et al. were able to show that both cell types release fewer molecules per event at lower temperatures $\left(21^{\circ} \mathrm{C}\right.$ for mast cells and $22{ }^{\circ} \mathrm{C}$ for chromaffin cells $)$ than at a temperature of $37^{\circ} \mathrm{C}$. The release events for both cell types were also found to display slower kinetics, as demonstrated by the half width of the peaks, at the lower temperature condition as well as a lower release frequency (Pihel et al. 1996). Haynes et al. used carbon fiber amperometry to study exocytotic release from chromaffin cells at temperatures ranging from 25 to $37^{\circ} \mathrm{C}$ (Haynes et al. 2007). By stimulating with potassium or barium they were able to study release from readily releasable and reserve vesicles, respectively. Their findings, in agreement with the study by Pihel et al. showed that decreasing the temperature from physiological conditions significantly decreases the average amount of catechol released per event. This was found to be true for stimulation with both $\mathrm{K}^{+}$and $\mathrm{Ba}^{2+}$, with a decrease in molecules released of approximately $6 \times 10^{4}{ }^{\circ} \mathrm{C}^{-1}$. In addition to smaller amounts released the release events were found to be kinetically slower as indicated by significantly increased peak half widths, as well as a decrease in release frequency as the temperature was lowered. The authors argued that the fact the half width of the events increases as the amount release decreases is evidence for a less efficient extrusion of the vesicular catecholamine as opposed to a loss of stored transmitter molecules. They suggested that this lowered extrusion efficiency at lower temperature is due to the temperature dependence of the behavior of Chromogranin A, which has been shown to become more disordered at higher temperatures. However, what is evident here is that the amount released was altered and for this to occur, partial release of the contents had to be occurring.

\subsection{External pH}

A change in external $\mathrm{pH}$ has been shown to rapidly alter the amount of catecholamine released from bovine adrenal medullary cells. These cells were shown to release more transmitter molecules per event at $\mathrm{pH} 8 \cdot 2$ than at $\mathrm{pH} 7.4$ when release was stimulated by $\mathrm{Ba}^{2+}$ in a $\mathrm{Ca}^{2+}$ free solution. The authors argued that this flexibility in amount released is based on the degree of vesicular matrix dissociation in the dense core vesicle. The dissociation is suggested to be enhanced when the $\mathrm{H}^{+}$gradient over the fusion pore is increased, leading to a larger amount released of the total vesicle content when the $\mathrm{pH}$ difference between vesicle and cell exterior is greater (Jankowski et al. 1994; Yoo \& Lewis, 1995). Although not explicitly stated by the authors, the dense core dissociation mechanism assumes partial release as this mechanism does not affect the amount contained in an individual vesicle. 


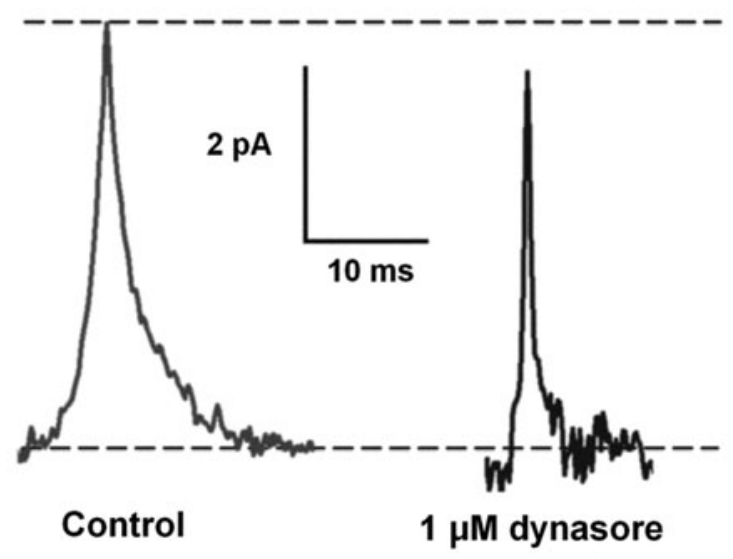

Fig. 12. Average amperometric peaks obtained from the control and $1 \mu \mathrm{M}$ dynasore treatments. Amperometry of exocytotic events from single PC12 cells (Trouillon \& Ewing, 2013). The working electrode was held at $+700 \mathrm{mV}$ versus an $\mathrm{Ag} / \mathrm{AgCl}$ reference electrode using an Axon $200 \mathrm{~B}$ potentiostat. The output was filtered at $2 \cdot 1 \mathrm{kHz}$ using a Bessel filter and digitized at $5 \mathrm{kHz}$.

\section{Regulation of open and closed exocytosis}

\subsection{Evidence that dynamin regulates the opening of the fusion pore}

If exocytosis is open and closed, then what regulates the process beyond formation of the fusion pore? Dynamin is a candidate for part of this process. Dynamin is a GTPase mechanochemical enzyme involved in the late steps of endocytosis, where it separates the endocytotic vesicle from the cell membrane. However, several recent reports have emphasized its role in exocytosis. In this case, dynamin appears to contribute to the control of the exocytotic pore, and possibly directly influencing the efflux of neurotransmitters.

Dynasore is a selective inhibitor of the GTPase activity of dynamin and this has been used to investigate the role of dynamin in exocytosis with amperometric detection (Trouillon \& Ewing, 2013). These experiments (Fig. 12) revealed that dynasore inhibits exocytosis in a dose-dependent manner. Analysis of the exocytotic peaks showed that the inhibition of the GTPase activity of dynamin led to shorter, smaller events. This observation is in good agreement with results published recently from near field microscopy observation of pore dynamics, where the GTPase activity of dynamin was inhibited by dynasore or mutations (Anantharam et al. 2011). The duration of the feet is also reduced, and the constraining effect of the pore is larger on the decaying part of the peak. In terms of pore dynamics, this appears to indicate that the fusion pore opens and closes faster, and that its maximum opening radius is decreased, after dynasore. Thus it has been proposed that dynamin is involved in regulating the duration and kinetics of the open and closed version of exocytotic release. The role of dynamin in endocytosis is thought to be tube closure. In exocytosis, dynamin appears to contribute to the dilation and stability of the pore.

In the case of open and closed exocytosis, it appears that the dynamin complex contributes to supporting, or framing, the nanotube formed between the vesicle and the membrane. In this case, the pore does not follow its intrinsic kinetics, but is apparently controlled by the dynamin complex. The longer $t_{\text {foot }}$ in presence of the fully active dynamin might indicate that the foot feature corresponds to the formation of the dynamin assembly and the initiation of GTPase activity, followed by the pore expansion. This concept, supported by several studies (Berberian et al. 2009; Burgoyne et al. 2001; Fulop et al. 2008; Guzman et al. 2007; Wang \& Richards, 2011) suggests that the mechanochemical activity of dynamin is involved in exocytosis, and that this activity relies on its GTPase properties.

\subsection{Evidence that actin regulates the closing of the fusion pore}

A key question in open and closed exocytosis, is what closes the pore? The effect of latrunculin A, an inhibitor of actin cross-linking, on exocytosis in PC12 cells has been investigated, again with single cell amperometry to examine if the actin cytoskeleton might be involved in regulating exocytosis, especially by mediating the constriction of the pore (Trouillon \& Ewing, 2014). In an open and closed release mode, actin could actually control the fraction of neurotransmitter molecules released by the vesicle. Following application of latrunculin A to PC12 cells, a $40 \%$ increase of the median of the amount released is observed (51\% increase of the mean release). The frequency of large events ( $>200000$ molecules released) is also increased. One of the main variations in the peak characteristics after the latrunculin A treatment is 


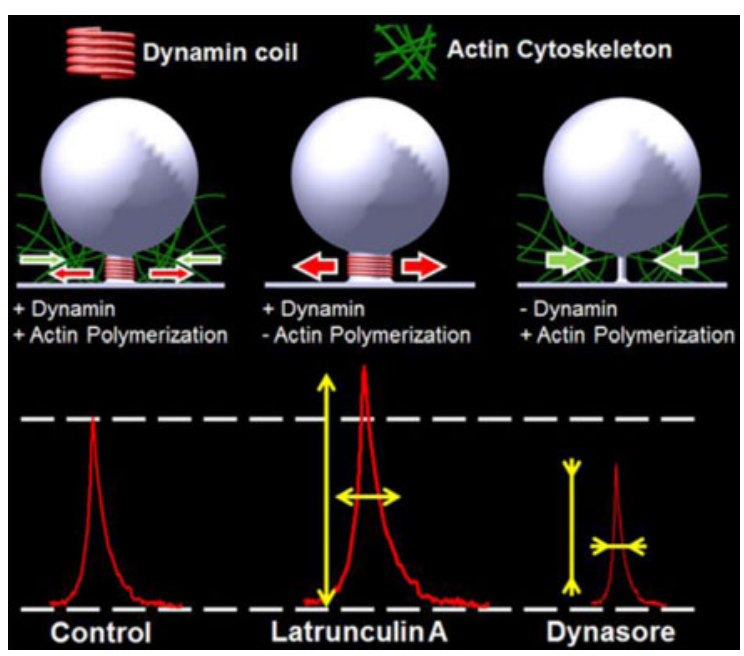

Fig. 13. Proposed scheme for the contribution of dynamin and actin to exocytosis. Actin appears to force the closing of the pore, whereas dynamin promotes its opening (Trouillon \& Ewing, 2014). The resulting amperometric spikes are presented in the bottom portion of the figure (not drawn to scale). Exposure to latrunculin A induces higher, wider peaks, and inhibition of the GTPase activity of dynamin with the inhibitor dynasore induces shorter, narrower peaks.

the observed increase in peak fall time. The decaying part of the peak is expected to contain some information about the closing of the fusion pore in open and closed exocytosis (Allersma et al. 2004). In this case, the increased fall time is evidence that the inhibition of actin polymerization increases the lifetime of the pore, and slows down the collapse of the pore. Thus, it is argued that actin is an important factor in the closing of the lipid pore. These findings strongly support the theory of partial release at PC12 cells, and that actin is involved in regulating the amount of vesicular content released during open and closed exocytosis.

Interestingly, actin is known to interact with several membrane-shaping chemical motors, such as dynamin (Gu et al. 2010; Orth \& McNiven, 2003; Pelkmans et al. 2002). Together the two proteins appear to be part of the regulation of the fusion pore dynamics in a competitive process between different mechanochemical transducers, as summarized in Fig. 13 (Trouillon \& Ewing, 2014). In this case, dynamin, at least in part, promotes the opening of the pore, and actin its closure. It appears likely that actin and dynamin complement each other in balancing and controlling release during an open and closed exocytosis process. The composition of the lipid membrane also plays a critical part. However, this list is not exhaustive, as other polymers, proteins or lipid structures clearly might contribute to this highly dynamic and competitive process.

\section{Summary of the evidence for open and closed exocytosis}

The evidence for open and closed exocytosis as the primary mechanism of full exocytosis is mostly based on amperometric measurements and overall it is apparent that the different methods measure different aspects of release and perhaps different release events. It is important to note that for whole cell patch clamp, one measures exocytosis over an entire cell, whereas for amperometry, a $5-\mu \mathrm{m}$ electrode covers perhaps $5 \%$ of a chromaffin or PC12 cell. TIRFM measurements look at the membrane along the bottom of the cell. The fact that there are many more events measured at a typical cell with amperometry, a method capable of measuring faster events, offers the possibility that amperometry methods primarily measure the faster open and closed events and that TIRFM and patch clamp primarily measure the full distention events. There appear to be fewer of these and searching for larger events with amperometry suggests that under control conditions about $2 \%$ of those at PC12 cells are the larger full distention events representing complete release of the vesicle content.

The following is the key evidence suggesting partial release during exocytosis is dominant.

\subsection{The majority of exocytotic events are followed by immediate endocytosis}

Using a Quartz Crystal Microbalance with Dissipation, Cans et al. were able to study both exocytosis and endocytosis simultaneously for a population of cells. 


\subsection{The total content of a secretory vesicle is greater than the amount released in PC12 cells}

The amount released from individual vesicles containing electroactive neurotransmitters such as dopamine can be measured through electrochemical methods. Work in the Ewing laboratory compared conventional amerometric measurements of exocytosis with electrochemical cytometry of vesicle contents for the same line of cells. Comparative quantification of transmitters released during individual exocytosis events, using amperometry, and the total contents of individual vesicles, using cytometry at PC12 cells suggested that approximately 40\% of the total dopamine content was released during a single exocytosis event. Partial exocytosis is not only possible, but it is highly likely that it is the primary mode of exocytosis with only a small number of amperometric events releasing large quantities of dopamine in full ejection of the vesicle content.

\subsection{Release appears to be only a fraction of vesicle content in mammalian brains}

Electrochemical cytometry in live animal models results in vesicular contents that are much larger than what is apparently released. In fact, the data suggest that an even smaller fraction of transmitter is released from mammalian brain cells.

\subsection{Impact cytometry of adrenal cell vesicles shows a significantly larger catecholamine content then that released}

A new version of electrochemical cytometry has been used to confirm comparative measurements of catecholamines, now in chromaffin cell vesicles, and it is significantly larger than that released.

\subsection{Impact cytometry has been used to measure vesicle content in live cells and this quantity is greater than the amount released}

Nanometer electrodes have been used for intracellular measurements of vesicle content in live PC12 cells, and using electrodes of identical signal to noise the released transmitter was measured to be approximately $65 \%$ of the total content, confirming the results from early cytometry experiments.

\subsection{Full distention of the vesicle is not necessary for the measured amperometric current during exocytotic release}

Amatore et al. have studied the maximum aperture angle necessary for amperometric detection of complete release of vesicle contents during exocytosis and found that the vesicle only needs to open to approximately $10^{\circ}$ in order to release its contents, as opposed to completely opening as part of the cell membrane, to generate the current observed in the amperometric measurements of exocytosis.

\subsection{Fast changes in cell environment alter the amount of messenger released}

Temperature, osmotic stress and other environmental stresses can change the amount of exocytosis faster than would be expected to change vesicle content. The change in the amount of release is consistent with a partial release mechanism.

\subsection{Patch amperometry measurements reveal a greater amount of release}

When an electrode is placed inside a patch clamp pipette, and the pipette used to pull on a cell membrane, the resulting amperometric measurement of exocytosis reveals that considerably larger amounts are released. It appears that the tension on the membrane caused by suction from the pipette causes the vesicles to undergo full distention and release all their contents.

\subsection{Postspike 'feet' are observed with an amperometric spike}

Features have been detected at the end of an exocytosis spike for some of exocytosis events. These post spike features have lower current than pre-spike features, but a similar total charge was released indicating the closing of the vesicle after a partial release of contents. Taken together, the presence of pre-spike and post spike features demonstrates that a stable fusion pore exists not only before, but also after the release event that has been previously thought to be complete exocytosis, and thus suggests again that the pore closes.

It appears that when amperometry is used to measure exocytosis events, nearly all the events measured are from open and closed exocytosis. This is distinguished from kiss-and-run exocytosis in that what we measure as predominant exocytosis events with amperometric methods is open and closed, whereas kiss-and-run is a much smaller opening of the fusion pore (flicker) that releases a very small amount of the vesicle content. In addition to the predominant open and closed events, perhaps a few percent of the events monitored with amperometry are from full distention of the vesicle during the fusion process. These few events might be those that are measured by patch capacitance measurements and TIRFM. 
Amperometry typically measures from about 5\% of the cell surface and yet there are about 10-20 times more exocytosis events observed compared with patch and TIRFM methods. Furthermore, these larger events are slower and are more amenable to measurement by those methods. If exocytosis is open and closed, then the exocytosis process can be regulated at the level of single events in terms of the percentage and the rate of released messengers. This avails new pharmacological targets and can be involved in plasticity.

\section{Acknowledgements}

We acknowledge many coworkers, who have come before and whose works we cite in this review. We acknowledge the support from the European Research Council (ERC Advanced Grant), the Knut and Alice Wallenberg Foundation in Sweden, the Swedish Research Council (VR), and the U.S. National Institutes of Health (NIH).

\section{References}

Akopova, I., Tatur, S., GrygorczyK, M., Luchowski, R., Gryczynski, I., Gryczynski, Z., Borejdo, J. \& Grygorczyk, R. (2011). Imaging exocytosis of ATP-containing vesicles with TIRF microscopy in lung epithelial A549 cells. Purinergic Signalling 8, 59-70.

Albillos, A., Dernick, G., Horstmann, H., Almers, W., De Toledo, G. A. \& Lindau, M. (1997). The exocytotic event in chromaffin cells revealed by patch amperometry. Nature $389,509-512$.

Alés, E., Tabares, L., Poyato, J. M., Valero, V., Lindau, M. \& De Toledo, G. A. (1999). High calcium concentrations shift the mode of exocytosis to the kiss-and-run mechanism. Nature Cell Biology 1, 40-44.

Allersma, M. W., Wang, L., Axelrod, D. \& Holz, R. W. (2004). Visualization of regulated exocytosis with a granule membrane probe using total internal reflection microscopy. Molecular Biology of the Cell 15, 4658-4668.

Amatore, C., Arbault, S., Bonifas, I., Bouret, Y., Erard, M., Ewing, A. G. \& Sombers, L. A. (2005). Correlation between vesicle quantal size and fusion pore release in chromaffin cell exocytosis. Biophysical Journal 88, 4411-4420.

Amatore, C., Arbault, S., Bouret, Y., Guille, M., Lemaitre, F. \& Verchier, Y. (2006). Regulation of exocytosis in chromaffin cells by trans-insertion of lysophosphatidylcholine and arachidonic acid into the outer leaflet of the cell membrane. ChemBioChem 7, $1998-2003$.

Amatore, C., Arbault, S., Bonifas, I., Guille, M., Lemaittre, F. \& Verchier, Y. (2007). Relationship between amperometric pre-spike feet and secretion granule composition in chromaffin cells: an overview. Biophysical Chemistry 129, 181-189.

Amatore, C., Arbault, S., Bonifas, I. \& Guille, M. (2009). Quantitative investigations of amperometric spike feet suggest different controlling factors of the fusion pore in exocytosis at chromaffin cells. Biophysical Chemistry 143, 124-131.

Amatore, C., Oleinick, A. I. \& Svir, I. (2010). Reconstruction of aperture functions during full fusion in vesicular exocytosis of neurotransmitters. ChemPhysChem 11, 159-174.

Anantharam, A., Bittner, M. A., Aikman, R. L., Stuenkel, E. L., Schid, S. L., Axelrod, D. \& Holz, R. W. (2011). A new role for the dynamin GTPase in the regulation of fusion pore expansion. Molecular Biology of the Cell 22, 1907-1918.

Aravanis, A. M., Pyle, J. L., Harata, N. C. \& Tsien, R. W. (2003). Imaging single synaptic vesicles undergoing repeated fusion events: kissing, running, and kissing again. Neuropharmacology 45, 797-813.

Axelrod, D. (1989) Fluorescence Microscopy of Living Cells in Culture, Part B: Total internal reflection fluorescence flow cytometry (eds. L. TAYLoR \& Y-L. WANG), pp. 245-269. San Diego: Academic Press.

Barg, S., Olofsson, C. S., Schriever-Abeln, J., Wendt, A., Gebre-Medhin, S., Renstrom, E. \& Rorsman, P. (2002). Delay between fusion pore opening and peptide release from large dense-core vesicles in neuroendocrine cells. Neuron 33, 287-299.

Becherer, U., Pasche, M., Nofal, S., Hof, D., Matti, U. \& Rettig, J. (2007). Quantifying exocytosis by combination of membrane capacitance measurements and total internal reflection fluorescence microscopy in chromaffin cells. PLoS ONE 6, 1-6.

Berberian, K., Torres, A. J., Fang, Q., Kisler, K. \& Lindau, M. (2009). F-actin and myosin II accelerate catecholamine release from chromaffin granules. Journal of Neuroscience 29, 863-870.

BetZ, W. \& Bewick, G. (1992). Optical analysis of synaptic vesicle recycling at the frog neuromuscular junction. Science 255, $200-203$.

BEтZ, W. J., MAO, F. \& BЕWiсK, G. S. (1992). Activity-dependent fluorescent staining and destaining of living vertebrate motor nerve terminals. Journal of Neuroscience 12, 363-375.

Betz, W. J., Maо, F. \& Sмith, C. B. (1996). Imaging exocytosis and endocytosis. Current Opinion in Neurobiology 6, 365-371.

Borges, R., Travis, E. R., Hochstetler, S. E. \& Wightman, R. M. (1997). Effects of external osmotic pressure on vesicular secretion from bovine adrenal medullary cells. Journal of Biological Chemistry 272, 8325-8331.

Brown, E. M., Pazoles, C. J., Creutz, C. E., Aurbach, G. D. \& Pollard, H. B. (1978). Role of anions in parathyroid hormone release from dispersed bovine parathyroid cells. Proceedings of the National Academy of Sciences of the United States of America 75, 876-880.

BRUNS, D. \& JAHN, R. (1995). Real-time measurement of transmitter release from single synaptic vesicles. Nature 377, 62-65.

Burgoyne, R. D., Fisher, R. J., Graham, M. E., Haynes, L. P. \& Morgan, A. (2001). Control of membrane fusion dynamics during regulated exocytosis. Biochemical Society Transactions 29, 467-472.

Cahill, P. S., Walker, Q. D., Finnegan, J. M., Mickelson, G. E., Travis, E. R. \& Wightman, R. M. (1996). Microelectrodes for the measurement of catecholamines in biological systems. Analytical Chemistry 68, 3180-3186. 
Cans, A.-S., Höök, F., Shupliakov, O., Ewing, A. G., Eriksson, P. S., Brodin, L. \& Orwar, O. (2001). Measurement of the dynamics of exocytosis and vesicle retrieval at cell populations using a quartz crystal microbalance. Analytical Chemistry 73, 5805-5811.

Chow, R. H., Von Ruden, L. \& Neher, E. (1992). Delay in vesicle fusion revealed by electrochemical monitoring of single secretory events in adrenal chromaffin cells. Nature 356, 60-63.

Chow, R. H., Klingauf, J., Heinemann, C., Zucker, R. S. \& Neher, E. (1996). Mechanisms determining the time course of secretion in neuroendocrine cells. Neuron 16, 369-376.

Ciolkowski, E. L., Cooper, B. R., Jankowski, J. A., Jorgenson, J. W. \& Wightman, R. M. (1992). Direct observation of epinephrine and norepinephrine cosecretion from individual adrenal medullary chromaffin cells. Journal of the American Chemical Society 114, $2815-2821$.

Couteaux, R. \& Pecot-Dechavassine, M. (1970). Vésicules synaptiques et poches au niveau des 'zones actives' de la jonction neuromusculaire. Comptes Rendus de 'Académie des Sciences (Paris) 271, 2346-2349.

Del Castillo, J. \& Katz, B. (1954). Quantal components of the end-plate potential. Journal of Physiology 124, 560-573.

Del Castillo, J. \& Katz, B. (1957). La base 'quantale' de la transmission neuromusculaire, Microphysiologie comparée des éléments excitables. CNRS Paris 67, 245-258.

De Toledo, G. A., Fernandez-Chacon, R. \& Fernandez, J. M. (1993). Release of secretory products during transient vesicle fusion. Nature 363, 554-558.

Dernick, G., Gong, L.-W., Tabares, L., De Toledo, G. A. \& Lindau, M. (2005). Patch amperometry: high-resolution measurements of singlevesicle fusion and release. Nature Methods 2, 699-708.

Dunevall, J., Fathali, H., Najafinobar, N., Lovric, J., WigströM, J., Cans, A.-S. \& Ewing, A. G. (2015). Characterizing the catecholamine content of single mammalian vesicles by collision-adsorption events at an electrode. Journal of the American Chemical Society 137, 4344-4346.

Elhamdani, A., Azizi, F. \& Artalejo, C. R. (2006). Double patch clamp reveals that transient fusion (kiss-and-run) is a major mechanism of secretion in calf adrenal chromaffin cells: high calcium shifts the mechanism from kiss-and-run to complete fusion. Journal of Neuroscience 26, 3030-3036.

Englert, D. F. \& Perlman, R. L. (1981). Permeant anions are not required for norepinephrine secretion from pheochromocytoma cells. Biochimica et Biophysica Acta 674, 136-143.

FAtT, P. \& KATZ, B. (1952). Spontaneous subthreshold activity at motor nerve endings. Journal of Physiology 117, 109-128.

Fernandez, J. M., Neher, E. \& Gomperts, B. D. (1984). Capacitance measurements reveal stepwise fusion events in degranulating mast-cells. Nature 312, 453-455.

Fernández-Peruchena, C., Navas, S., Montes, M. A. \& Állvarez De Toledo, G. (2005). Fusion pore regulation of transmitter release. Brain Research Reviews 49, 406-415.

Finkelstein, A., Zimmerberg, J. \& Cohen, F. S. (1986). Osmotic swelling of vesicles: its role in the fusion of vesicles with planar phospholipid bilayer membranes and its possible role in exocytosis. Annual Review of Physiology 48, 163-174.

Foley, K., Boguslavsky, S. \& Klip, A. (2011). Endocytosis, recycling, and regulated exocytosis of glucose transporter 4. Biochemistry 50, 3048-3061.

Frolov, V. A., Lizunov, V. A., Dunina-Barkovskaya, A. Y., Samsonov, A. V. \& Zimmerberg, J. (2003). Shape bistability of a membrane neck: a toggle switch to control vesicle content release. Proceedings of the National Academy of Sciences of the United States of America 100, 8698-8703.

Fulop, T., Doreian, B. \& Smith, C. (2008). Dynamin I plays dual roles in the activity-dependent shift in exocytic mode in mouse adrenal chromaffin cells. Archives of Biochemistry and Biophysics 477, 146-154.

Ge, S. C., Woo, E., White, J. G. \& Haynes, C. L. (2011). Electrochemical measurement of endogenous serotonin release from human blood platelets. Analytical Chemistry 83, 2598-2604.

Gu, C., Yaddanapudi, S., Weins, A., Osborn, T., Reiser, J., Pollak, M., Hartwig, J. \& Sever, S. (2010). Direct dynamin-actin interactions regulate the actin cytoskeleton. EMBO Journal 29, 3593-3606.

Guzman, R., Bolanos, P., Delgado, A., Rojas, H., Dipolo, R., Caputo, C. \& Jaffe, E. (2007). Depolymerisation and rearrangement of actin filaments during exocytosis in rat peritoneal mast cells: involvement of ryanodine-sensitive calcium stores. European Journal of Physiology 454, 131-141.

Hampton, R. Y. \& Holz, R. W. (1983). Effects of changes in osmolality on the stability and function of cultured chromaffin cells and the possible role of osmotic forces in exocytosis. Journal of Cell Biology 96, 1082-1088.

Haynes, C. L., Siff, L. N. \& Wightman, R. M. (2007). Temperature-dependent differences between readily releasable and reserve pool vesicles in chromaffin cells. Biochimica et Biophysica Acta (BBA)-Molecular Cell Research 1773, 728-735.

HE, L., WU, X.-S., Mohan, R. \& WU, L.-G. (2006). Two modes of fusion pore opening revealed by cell-attached recordings at a synapse. Nature 444, 102-105.

Heuser, J. \& Miledi, R. (1971). Effect of lanthanum ions on function and structure of frog neuromuscular junctions. Proceedings of the Royal Society of London. Series B. Biological Sciences 179, 247-260.

Heuser, J. \& ReESE, T. (1981). Structural changes after transmitter release at the frog neuromuscular junction. Journal of Cell Biology 88 , $564-580$.

Holt, M., Cooke, A., Neef, A. \& Lagnado, L. (2004). Omega shape- high mobility of vesicles supports continuous exocytosis at a ribbon synapse. Current Biology 14, 173-183.

Holz, R. W. (1986). The role of osmotic forces in exocytosis from adrenal chromaffin cells. Annual Review of Physiology 48, 175-189. 
Izquierdo, L. A., Barros, D. M., Vianna, M. R. M., Coitinho, A., Silva, T. D. E., Choi, H., Moletta, B., Medina, J. H. \& Izquierdo, I. (2002). Molecular pharmacological dissection of short- and long-term memory. Cellular and Molecular Neurobiology 22, $269-287$.

Jahn, R. \& Sudhof, T. C. (1994). Synaptic vesicles and exocytosis. Annual Review of Neuroscience 17, 219-246.

Jankowski, J. A., Finnegan, J. M. \& Wightman, R. M. (1994). Extracellular ionic composition alters kinetics of vesicular release of catecholamines and quantal size during exocytosis at adrenal medullary cells. Journal of Neurochemistry 63, 1739-1747.

Jin, H., Heller, D. A. \& Strano, M. S. (2008). Single-particle tracking of endocytosis and exocytosis of single-walled carbon nanotubes in NIH-3T3 cells. Nano Letters 8, 1577-1585.

KILIC, G. (2002). Exocytosis in bovine chromaffin cells: studies with patch-clamp capacitance and FM1-43 fluorescence. Biophysical Journal 83, 849-857.

Kops, S. K., Theoharides, T. C., Cronin, C. T., Kashgarian, M. G. \& Askenase, P. W. (1990). Ultrastructural characteristics of rat peritoneal mast cells undergoing differential release of serotonin without histamine and without degranulation. Cell and Tissue Research 262, 415-424.

Lawson, D., Raff, M. C., Gomperts, B., Fewtrell, C. \& Gilula, N. B. (1977). Molecular events during membrane fusion: a study of exocytosis in rat peritoneal mast cells. Journal of Cell Biology 72, 242-259.

Leszczyszyn, D. J., Jankowski, J. A., Viveros, O. H., Diliberto, E., Near, J. \& Wightman, R. (1990). Nicotinic receptor-mediated catecholamine secretion from individual chromaffin cells. Chemical evidence for exocytosis. Journal of Biological Chemistry 265, 14736-14737.

Li, X., Majdi, S., Dunevall, J., Fathali, H. \& Ewing, A. G. (2015). Quantitative measurement of transmitters in individual vesicles in the cytoplasm of single cells with nanotip electrodes. Angewandte Chemie International Edition 54, 11978-11982.

Lin, Y., Trouillon, R., Svensson, M. I., Keighron, J. D., Cans, A.-S. \& Ewing, A. G. (2012). Carbon-ring microelectrode arrays for electrochemical imaging of single cell exocytosis: fabrication and characterization. Analytical Chemistry 84, 2949-2954.

Mellander, L. J., Trouillon, R., Svensson, M. I. \& Ewing, A. G. (2012). Amperometric post spike feet reveal most exocytosis is via extended kiss-and-run fusion. Scientific Reports 2, 1-6.

MiLEDI, R. (1966). Strontium as a substitute for calcium in the process of transmitter release at the neuromuscular junction. Nature 212, 1233-1234.

Mohrmann, R., De Wit, H., Verhage, M., Neher, E. \& Sørensen, J. B. (2010). Fast vesicle fusion in living cells requires at least three SNARE complexes. Science 330, 502-505.

Montesinos, M. S., Machado, J. D., Camacho, M., Diaz, J., Morales, Y. G., De la Rosa, D. A., Carmona, E., Castaneyra, A., Viveros, O. H., O'Connor, D. T., MAhAtA, S. K. \& Borges, R. (2008). The crucial role of chromogranins in storage and exocytosis revealed using chromaffin cells from chromogranin A null mouse. Journal of Neuroscience 28, 3350-3358.

Neher, E. \& Marty, A. (1982). Discrete changes of cell membrane capacitance observed under conditions of enhanced secretion in bovine adrenal chromaffin cells. Proceedings of the National Academy of Sciences of the United States of America 79, 6712-6716.

Neher, E., Sakmann, B. \& Steinbach, J. H. (1978). The extracellular patch clamp: a method for resolving currents through individual open channels in biological membranes. Pflugers Arch - European Journal of Physiology 375, 219-228.

Омiatek, D. M., Santillo, M. F., Heien, M. L. \& Ewing, A. G. (2009). Hybrid capillary-microfluidic device for the separation, lysis, and electrochemical detection of vesicles. Analytical Chemistry 81, 2294-2302.

Omiatek, D. M., Dong, Y., Heien, M. L. \& Ewing, A. G. (2010). Only a fraction of quantal content is released during exocytosis as revealed by electrochemical cytometry of secretory vesicles. ACS Chemical Neuroscience 1, 234-245.

OmiateK, D. M., Bressler, A. J., Cans, A.-S., Andrews, A. M., Heien, M. L. \& Ewing, A. G. (2013). The real catecholamine content of secretory vesicles in the CNS revealed by electrochemical cytometry. Scientific Reports 3, 1-6.

Orth, J. D. \& Mcniven, M. A. (2003). Dynamin at the actin-membrane interface. Current Opinion in Cell Biology 15, 31-39.

Pelkmans, L., Pứntener, D. \& Helenius, A. (2002). Local actin polymerization and dynamin recruitment in SV40-induced internalization of caveolae. Science 296, 535-539.

Pihel, K., Schroeder, T. J. \& Wightman, R. M. (1994). Rapid and selective cyclic voltammetric measurements of epinephrine and norepinephrine as a method to measure secretion from single bovine adrenal medullary cells. Analytical Chemistry 66, 4532-4537.

Pihel, K., Travis, E. R., Borges, R. \& Wightman, R. M. (1996). Exocytotic release from individual granules exhibits similar properties at mast and chromaffin cells. Biophysical Journal 71, 1633.

Pollard, H. B., Tack-Goldman, K., Pazoles, C. J., Creutz, C. E. \& Shulman, N. R. (1977). Evidence for control of serotonin secretion from human platelets by hydroxyl ion transport and osmotic lysis. Proceedings of the National Academy of Sciences of the United States of America 74, 5295-5299.

Pothos, E. N., Davila, V. \& Sulzer, D. (1998). Presynaptic recording of quanta from midbrain dopamine neurons and modulation of the quantal size. Journal of Neuroscience 18, 4106-4118.

Pothos, E. N., Larsen, K. E., Krantz, D. E., Liu, Y.-J., Haycock, J. W., Setlik, W., Gershon, M. D., Edwards, R. H. \& Sulzer, D. (2000). Synaptic vesicle transporter expression regulates vesicle phenotype and quantal size. Journal of Neuroscience 20, 7297-7306.

Schmoranzer, J., Goulian, M., Axelrod, D. \& Simon, S. M. (2000). Imaging constitutive exocytosis with total internal reflection fluorescence microscopy. Journal of Cell Biology 149, 23-31.

Schroeder, T. J., Borges, R., Finnegan, J. M., Pihel, K., Amatore, C. \& Wightman, R. M. (1996). Temporally resolved, independent stages of individual exocytotic secretion events. Biophysical Journal 70, 1061-1068.

Segovia, M., Alés, E., Montes, M. A., Bonifas, I., Jemal, I., Lindau, M., Maximov, A., Südhof, T. C. \& De Toledo, G. A. (2010). Push-and-pull regulation of the fusion pore by synaptotagmin-7. Proceedings of the National Academy of Sciences of the United States of America 107, 19032-19037. 
Sesaki, H. \& Ogihara, S. (1997). Protrusion of cell surface coupled with single exocytotic events of secretion of the slime in Physarum plasmodia. Journal of Cell Science 110, 809-818.

Sмith, C. B. \& Betz, W. J. (1996). Simultaneous independent measurement of endocytosis and exocytosis. Nature 380, 531-534.

Sombers, L. A., Hanchar, H. J., Colliver, T. L., Wittenberg, N., Cans, A., Arbault, S., Amatore, C. A. \& Ewing, A. G. (2004). The effects of vesicular volume on secretion through the fusion pore in exocytotic release from PC12 cells. Journal of Neuroscience 24, $303-309$.

StaAl, R. G., Mosharov, E. V. \& Sulzer, D. (2004). Dopamine neurons release transmitter via a flickering fusion pore. Nature Neuroscience 7 , $341-346$.

Steyer, J. A., Horstmann, H. \& Almers, W. (1997). Transport, docking and exocytosis of single secretory granules in live chromaffin cells. Nature 388, 474-478.

Travis, E. R. \& Wightman, R. M. (1998). Spatio-temporal resolution of exocytosis from individual cells. Annual Review of Biophysics and Biomolecular Structure 27, 77.

Trouillon, R. \& Ewing, A. G. (2013). Amperometric measurements at cells support a role for dynamin in the dilation of the fusion pore during exocytosis. Chemphyschem 14, 2295-2301.

Trouillon, R. \& Ewing, A. G. (2014). Actin controls the vesicular fraction of dopamine released during extended kiss and run exocytosis. ACS Chemical Biology 9, 812-820.

TSAI, C.-C., YANG, C.-C., SHih, P.-Y., Wu, C.-S., ChEN, C.-D., PAN, C.-Y. \& CHEN, Y.-T. (2008). Exocytosis of a single bovine adrenal chromaffin cell: the electrical and morphological studies. Journal of Physical Chemistry B 112, 9165-9173.

Uchiyama, Y., Maxson, M. M., Sawada, T., Nakano, A. \& Ewing, A. G. (2007). Phospholipid mediated plasticity in exocytosis observed in PC12 cells. Brain Research 1151, 46-54

Vonruden, L. \& Neher, E. (1993). A ca-dependent early step in the release of catecholamines from adrenal chromaffin cells. Science 262, 1061-1065.

Wang, C.-T., Lu, J.-C., Bai, J., Chang, P. Y., Martin, T. F. J., Chapman, E. R. \& Jackson, M. B. (2003). Different domains of synaptotagmin control the choice between kiss-and-run and full fusion. Nature 424, 943-947.

WAng, J., BaO, N., PARIs, L. L., Geahlen, R. L. \& LU, C. (2008). Total internal reflection fluorescence flow cytometry. Analytical Chemistry 80, 9840-9844.

WANG, J. \& Richards, D. A. (2011). Spatial regulation of exocytic site and vesicle mobilization by the actin cytoskeleton. PLoS ONE 6, 1-6.

Wang, J., Trouillon, R., Dunevall, J. \& Ewing, A. G. (2014). Spatial resolution of single-cell exocytosis by microwell-based individually addressable thin film ultramicroelectrode arrays. Analytical Chemistry 86, 4515-4520.

Wightman, R. M. \& Haynes, C. L. (2004). Synaptic vesicles really do kiss and run. Nature Neuroscience 7, 321-322.

Wightman, R. M., Jankowski, J. A., Kennedy, R. T., Kawagoe, K. T., Schroeder, T. J., Leszczyszyn, D. J., Near, J. A., Diliberto, E. J. \& Viveros, O. H. (1991). Temporally resolved catecholamine spikes correspond to single vesicle release from individual chromaffin cells. Proceedings of the National Academy of Sciences of the United States of America 88, 10754-10758.

WU, L.-G., Hamid, E., Shin, W. \& Chiang, H.-C. (2014). Exocytosis and endocytosis: modes, functions, and coupling mechanisms. Annual Review of Physiology 76, 301-331.

Xia, X. F., Lessmann, V. \& Martin, T. F. J. (2009). Imaging of evoked dense-core-vesicle exocytosis in hippocampal neurons reveals long latencies and kiss-and-run fusion events. Journal of Cell Science 122, 75-82.

Yoo, S. H. \& LewIS, M. S. (1995). Thermodynamic study of the pH-dependent interaction of chromogranin A with an intraluminal loop peptide of the inositol 1, 4, 5-trisphosphate receptor. Biochemistry 34, 632-638.

Zenisek, D., Steyer, J.A. \& Almers, W. (2000). Transport, capture and exocytosis of single synaptic vesicles at active zones. Nature 406, 849-854.

ZhANG, J., XUE, R., ONG, W.-Y. \& ChEN, P. (2009a). Roles of cholesterol in vesicle fusion and motion. Biophysical Journal 97, 1371-1380.

Zhang, Q., CAO, Y.-Q. \& Tsien, R. W. (2007). Quantum dots provide an optical signal specific to full collapse fusion of synaptic vesicles. Proceedings of the National Academy of Sciences of the United States of America 104, 17843-17848.

Zhang, Q., LI, Y. \& Tsien, R. W. (2009b). The dynamic control of kiss-and-run and vesicular reuse probed with single nanoparticles. Science 323, 1448-1453.

Zhang, Z., Wu, Y., Wang, Z., Dunning, F. M., Rehfuss, J., Ramanan, D., Chapman, E. R. \& Jacksona, M. B. (2011). Release mode of large and small dense-core vesicles specified by different synaptotagmin isoforms in PC12 cells. Molecular Biology of the Cell 22, 2324-2336.

Zhou, Z., Misler, S. \& Chоw, R. H. (1996). Rapid fluctuations in transmitter release from single vesicles in bovine adrenal chromaffin cells. Biophysical Journal 70, 1543-1552.

Zimmerberg, J., Curran, M., Cohen, F. S. \& Brodwick, M. (1987). Simultaneous electrical and optical measurements show that membrane fusion precedes secretory granule swelling during exocytosis of beige mouse mast cells. Proceedings of the National Academy of Sciences of the United States of America 84, 1585-1589.

ZuCKER, R. S. (1996). Exocytosis: a molecular and physiological perspective. Neuron 17, 1049-1055. 